\title{
Two-Dimensional Adaptive Array Beamforming With Multiple Beam Constraints Using a Generalized Sidelobe Canceller
}

\author{
Ju-Hong Lee and Yung-Han Lee
}

\begin{abstract}
This paper deals with the problem of two-dimensional (2-D) adaptive array beamforming with multiple beam constraints (MBC) using a generalized sidelobe canceller (GSC). We present a method for the construction of signal blocking matrix required by the 2-D GSC. The resulting 2-D adaptive beamformer can provide almost the same performance as conventional 2-D adaptive beamformers based on a linearly constrained minimum variance (LCMV) criterion. The effectiveness of the proposed GSC is that the construction of the required signal blocking matrix requires only the computation of a few entries from analytical formulas. In comparison with conventional methods, the proposed technique gets rid of the computational complexity due to the eigendecomposition required for finding the 2-D signal blocking matrix. For dealing with the performance degradation due to coherent interference, we present a 2-D weighted spatial smoothing scheme to effectively alleviate the coherent jamming effect. Several simulation examples are provided for illustration and comparison.
\end{abstract}

Index Terms-Adaptive beamforming, generalized sidelobe canceller, multiple beam constraint.

\section{INTRODUCTION}

$\mathbf{R}$ ESEARCH regarding the design and analysis of adaptive array beamformers is widely focused on the situations of using an array with one-dimensional (1-D) scenario. In this case, the signal sources and the array elements are assumed to be coplanar. However, in many practical applications, there may exist different signals impinging on a 1-D array with different directions, but these signals possess the same angle with respect to the broadside of the 1-D array. However, a 1-D array can only distinguish the azimuth angle or the elevation angle in the absence of a number of interfering signals. Moreover, it is usually impossible to simply extend the principles and techniques of the 1-D case to deal with the mentioned problem. In these circumstances, we must resort to the use of adaptive beamforming with a two-dimensional (2-D) array scenario.

Adaptive beamforming using a generalized sidelobe canceller (GSC) has been widely considered due to its effectiveness and simplicity for achieving multiple linearly constrained beamforming [1], [2] and partially adaptive beamforming [3]-[6].

Manuscript received June 23, 2004; revised November 10, 2004. This work was supported by the National Science Council under Grant NSC92-2213-E002-033. The associate editor coordinating the review of this manuscript and approving it for publication was Dr. Fulvio Gini.

The authors are with the Department of Electrical Engineering, National Taiwan University, Taipei, 10617, Taiwan, R.O.C. (e-mail: juhong@cc.ee.ntu.edu.tw).

Digital Object Identifier 10.1109/TSP.2005.853155
Several other advantages of using a GSC-based 1-D adaptive beamformer over using conventional linearly constrained minimum variance (LCMV) 1-D adaptive beamformers are as follows. 1) The GSC-based adaptive beamformer is easily generalized to deal with main lobe derivative constraints of any order and combined temporal/spatial constraints [1]. 2) The GSC-based adaptive beamformer uses an unconstrained rather than a constrained algorithm to adapt the weights. It is able to adapt much faster [1]. 3) The GSC-based adaptive beamformer is less sensitive to coefficient quantization effects since the dynamic range of the signals in the adaptive portion of the beamformer is compressed [1]. 4) The GSC-based adaptive beamformer is a particularly suitable structure to use for studying the effect of random element amplitude and phase error effects on linearly constrained adaptive beamformers [19], [20]. However, there is very little work concerning adaptive beamforming based on GSC structure using a 2-D array. Recently, Salem et al. [7] presented the direct design of a GSC using a 2-D array to carry out 2-D adaptive beamforming with a main-beam constraint. Nevertheless, the 2-D blocking matrix $\boldsymbol{B}$ of the designed GSC is constructed from the formula $\boldsymbol{B}^{H} \boldsymbol{s}=\mathbf{o}$, where $s$ denotes the normalized elevation-azimuth steering vector of the desired signal, the superscript $H$ is constructed from the conjugate transpose, and $\mathbf{o}$ is constructed from the zero vector. Accordingly, the procedure for finding the required 2-D blocking matrix $\boldsymbol{B}$ is the direct extension of the 1-D case. This leads to the drawback that the computation complexity increases rapidly as the dimension of the 2-D array increases.

For many practical applications, such as satellite communications [8], an antenna array is required to possess the beamforming capability that receives more than one signal with specified gain constraints while suppressing all jammers. This goal can be achieved using an antenna array with a multiple-beam pattern [8], [9]. In the case of satellite communications using adaptive beamforming, short convergence time would be required for some applications like a target application. Yu [9] proposes an adaptive algorithm that finds an adaptive weight vector that is close to a desired quiescent beam pattern under a unit norm constraint on the weight. However, a sophisticated procedure is required to solve the resulting nonlinear optimization problem. To tackle this problem, Lee et al. [10] develop a technique based on Frost's algorithm [11] to provide adaptive beamforming with multiple-beam constraints (MBCs). However, this technique can only deal with the 1-D case.

In this paper, we present a technique to construct a GSCbased 2-D adaptive beamformer that possesses the capability 
to provide $\mathrm{MBC}$ as well as coherent jamming suppression. To achieve the first goal of using a GSC, we develop a mathematical formula for the construction of the 2-D signal blocking matrix $B$. The formula provides an analytical solution that finds the entries of the required $\boldsymbol{B}$ without the need for the computational complexity required by the conventional approach like [7]. Then, using the 2-D signal blocking matrix $\boldsymbol{B}$, we formulate the 2-D beamforming problem as finding such a quiescent weight matrix that the 2-D array output power is minimized subject to a set of a specified MBC. It is shown that an analytical solution for the resulting minimization problem can be easily obtained to satisfy the goal of providing MBC as well as jamming suppression. To achieve the second goal of effectively curing the problem due to the coherence between the desired signals and interferers, a 2-D weighted spatial smoothing scheme is presented. Several simulation examples are given to show the effectiveness of the proposed technique.

This paper is organized as follows. In Section II, we briefly describe the theory of 2-D adaptive beamforming using a GSC. Section III presents the technique for constructing a GSC-based 2-D adaptive beamformer with MBC. We introduce a 2-D weighted spatial smoothing scheme in Section IV. Several simulation examples are provided in Section $\mathrm{V}$ for illustration. We finally conclude the paper in Section VI.

\section{TWO-DIMENSIONAL ADAPTIVE ARRAY BEAMFORMING USING A GSC}

As shown in Fig. 1, a 2-D uniform planar array (UPA) with size $M \times N$ has sensors located on the 2-D X-Y plane at the positions $((m-1) \lambda / 2,(n-1) \lambda / 2)$ for $m=1,2, \ldots, M$ and $n=1,2, \ldots, N$, where $\lambda$ is the smallest signal wavelength of the signals with specified gain arrangements. Assume that the array phase center is set to the $\left(m_{0}, n_{0}\right)$ th sensor located at the position $\left.\left(\left(m_{0}-1\right) \lambda / 2\right),\left(n_{0}-1\right) \lambda / 2\right)$. Let the signal impinging on the 2-D array from the evaluation angle $\theta$ and azimuth angle $\phi$ yield a unit magnitude response and a phase response given by $\exp \left\{j\left[\left(m-m_{0}\right) u+\left(n-n_{0}\right) v\right]\right\}$ at the array sensor located at $((m-1) \lambda / 2,(n-1) \lambda / 2)$, where $j=\sqrt{-1}$, and $(u, v)=$ $(\pi \sin (\theta) \cos (\phi), \pi \sin (\theta) \sin (\phi)) . P$ narrowband and far-field signals are impinging on the 2-D UPA from $P$ distinct direction angles $\left(u_{i}, v_{i}\right)$ for $i=1,2, \ldots, P$. Hence, the data received by the $(m, n)$ th sensor can be written as

$$
\begin{array}{r}
x_{m n}(t)=\sum_{i=1}^{P} s_{i}(t) \exp \left\{j\left[\left(m-m_{0}\right) u_{i}+\left(n-n_{0}\right) v_{i}\right]\right\} \\
+z_{m n}(t)
\end{array}
$$

where $s_{i}(t)$ denotes the complex waveform of the $i$ th signal source, and $z_{m n}(t)$ signifies the spatially white sensor noise independent of $s_{i}(t)$. For simplicity, assume that the first $K$ signal sources are the desired signals with specified gain arrangements and direction angles $\left(u_{i}, v_{i}\right)$ for $i=1,2, \ldots, K$, whereas the other $P-K$ signals are interferers. From (1), we can express the data matrix received by the 2-D UPA as follows:

$$
\boldsymbol{X}(t) \sum_{i=1}^{P}\left[\boldsymbol{a}_{c}\left(u_{i}\right) \boldsymbol{a}_{r}\left(\boldsymbol{v}_{i}\right)^{T}\right] s_{i}(t)+Z(t)
$$

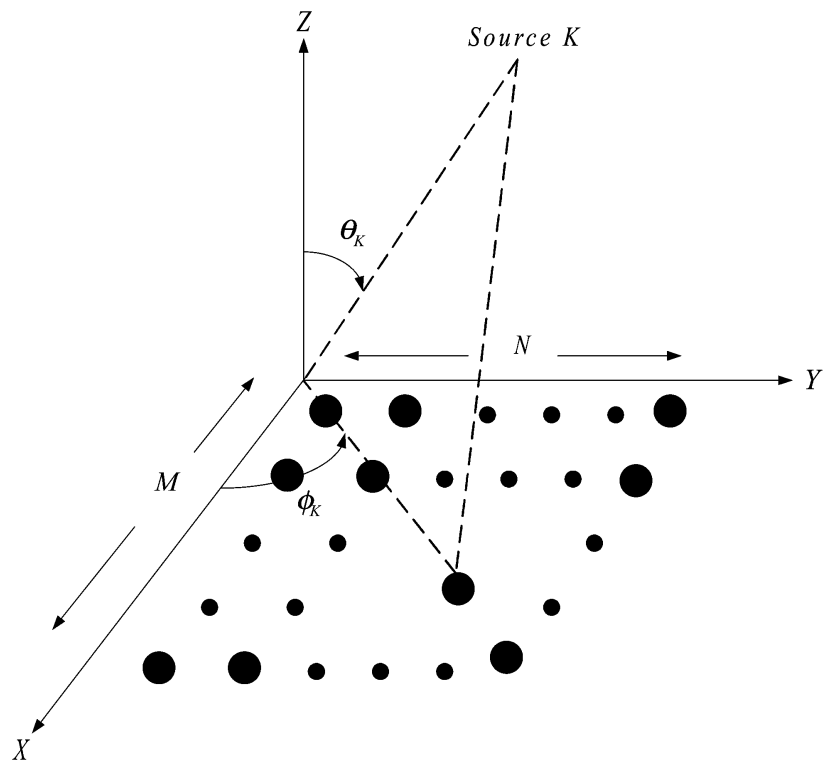

Fig. 1. Two-dimensional uniform planar array.

where $\boldsymbol{a}_{c}\left(u_{i}\right)=\left[\exp \left\{j\left(1-m_{0}\right) u_{i}\right\}, \exp \{j(2-\right.$ $\left.\left.\left.m_{0}\right) u_{i}\right\}, \ldots, \exp \left\{j\left(M-m_{0}\right) u_{i}\right\}\right]^{T}, \quad \boldsymbol{a}_{r}\left(v_{i}\right)=$ $\left[\exp \left\{j\left(1-n_{0}\right) v_{i}\right\}, \exp \left\{j\left(2-n_{0}\right) v_{i}\right\}, \ldots, \exp \left\{j\left(N-n_{0}\right) v_{i}\right\}\right]^{T}$, and $Z(t)$ denotes the noise matrix received by the 2-D UPA. The superscript $T$ represents the transpose operation. In vector form, we rewrite (2) as follows:

$$
\begin{aligned}
& \operatorname{vec}\{\boldsymbol{X}(t)\}=\boldsymbol{x}(t) \\
& =\left[x_{11}(t), x_{21}(t), \ldots, x_{M 1}(t)\right. \\
& \left.x_{12}(t), \ldots, x_{M 2}(t), \ldots, x_{M N}(t)\right]^{T} .
\end{aligned}
$$

Based on the property of the Kronecker product [12] $\operatorname{vec}\left\{\boldsymbol{F}_{1} \boldsymbol{F}_{2} \boldsymbol{F}_{3}^{T}\right\}=\left\{\boldsymbol{F}_{3} \otimes \boldsymbol{F}_{1}\right\} \operatorname{vec}\left\{\boldsymbol{F}_{2}\right\}$, where $\boldsymbol{F}_{i}$ are matrices with appropriate sizes, (3) can be written as follows:

$\operatorname{vec}\{\boldsymbol{X}(t)\}=\sum_{i=1}^{P} \boldsymbol{a}\left(u_{i}, v_{i}\right) s_{i}(t)+\operatorname{vec}\{Z(t)\}=\boldsymbol{A}_{s} \boldsymbol{s}(t)+\boldsymbol{z}(t)$

where the response vector of the $i$ th signal source $\boldsymbol{a}\left(u_{i}, v_{i}\right)=$ $\boldsymbol{a}_{r}\left(v_{i}\right) \otimes \boldsymbol{a}_{c}\left(u_{i}\right)$, the response matrix of the $P$ signal sources $\boldsymbol{A}_{s}=\left[\boldsymbol{a}\left(u_{1}, v_{1}\right) \boldsymbol{a}\left(u_{2}, v_{2}\right) \ldots \ldots \boldsymbol{a}\left(u_{P}, v_{P}\right)\right]$, and the signal source vector $\boldsymbol{s}(t)=\left[s_{1}(t), s_{2}(t), \ldots, s_{P}(t)\right]^{T}$. As a result, we have the correlation matrix of $\operatorname{vec}\{\boldsymbol{X}(t)\}$ given by

$$
\boldsymbol{R}_{x}=E\left\{\operatorname{vec}\{\boldsymbol{X}(t)\} \operatorname{vec}\{\boldsymbol{X}(t)\}^{H}\right\}=\boldsymbol{A}_{s} \boldsymbol{\Phi}_{s} A_{s}^{H}+\pi_{n} \boldsymbol{I}
$$

where $\boldsymbol{\Phi}_{s}=E\left\{\boldsymbol{s}(t) \boldsymbol{s}(t)^{H}\right\}$ denotes the correlation matrix of the signal sources, $\pi_{n}$ denotes the noise power, and $\boldsymbol{I}$ denotes the $M N \times M N$ identity matrix. Equation (5) reveals that $\boldsymbol{R}_{x}$ exhibits a block Toeplitz structure if the $P$ distinct signal sources are uncorrelated.

Consider the GSC structure shown by Fig. 2. It has been widely recognized that 1-D adaptive array beamforming using a GSC possesses the advantages of easy implementation and the assessment of the performance degradation due to steering or gain errors in array sensors. For the 2-D case with MBC, the output signal $d(t)$ of the upper branch is given by $d(t)=$ $\boldsymbol{w}_{q}^{H} \boldsymbol{x}(t)$. According to the theory of GSC [1], the weight vector 


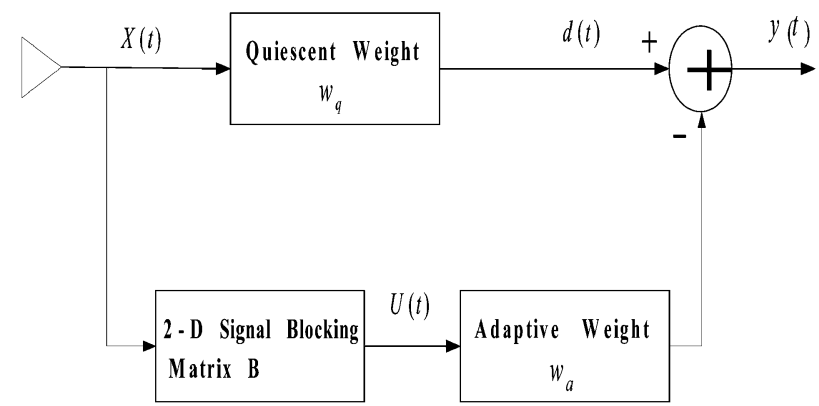

Fig. 2. Structure of a 2-D GSC.

$\boldsymbol{w}_{q}$ is the quiescent component of the overall weight vector of the GSC and used to realize the constrained weight subspace, which is spanned by the column vectors of the constraint matrix $C$. Hence, the design of $\boldsymbol{w}_{q}$ is asked to satisfy a set of linear constraints given by

$$
\boldsymbol{C}^{H} \boldsymbol{w}_{q}=f
$$

where $\boldsymbol{C}=\left[\boldsymbol{a}\left(u_{1}, v_{1}\right) \boldsymbol{a}\left(u_{2}, v_{2}\right) \ldots \boldsymbol{a}\left(u_{K}, v_{K}\right)\right]$ denotes the constraint matrix with size $M N \times K$, and $\boldsymbol{f}=\left[f_{1}, f_{2}, \ldots, f_{K}\right]^{T}$ is a $K \times 1$ gain vector containing the specified gain arrangements for the $K$ desired signal sources. Accordingly, $\boldsymbol{w}_{q}$ can be found as follows:

$$
\boldsymbol{w}_{q}=\boldsymbol{C}\left(\boldsymbol{C}^{H} \boldsymbol{C}\right)^{-1} \boldsymbol{f} .
$$

The sidelobe canceling branch is used for the realization of the unconstrained weight subspace that is complementarily orthogonal to the column space of the constraint matrix $C$. The 2-D signal blocking matrix $B$ is employed to block the $K$ desired signals from the received data vector $\boldsymbol{x}(t)$. It follows that $\boldsymbol{B}=\left[b_{i j}\right]$ with size $M N \times(M-K) N$ must be orthogonal to $\boldsymbol{C}$, i.e., $\boldsymbol{C}^{H} \boldsymbol{B}=\mathbf{o}$. The signal at the array output is given by $y(t)=\boldsymbol{w}_{q}^{H} \boldsymbol{x}(t)-\boldsymbol{w}_{a}^{H} \boldsymbol{B}^{H} \boldsymbol{x}(t)$. In this context, the adaptive weight $\boldsymbol{w}_{a}$ is the optimal solution for the unconstrained minimization problem:

$$
\text { Minimize } E\left\{|y(t)|^{2}\right\} \text {. }
$$

This leads to the fact that $\boldsymbol{w}_{a}$ is given by

$$
\boldsymbol{w}_{a}=\left(B^{H} \boldsymbol{R}_{x} B\right)^{-1} \boldsymbol{B}^{H} \boldsymbol{R}_{x} \boldsymbol{w}_{q} .
$$

The overall weight vector for the 2-D GSC-based adaptive beamformer becomes

$$
\boldsymbol{w}=\boldsymbol{w}_{q}-\boldsymbol{B} w_{a} .
$$

\section{Construction of 2-D Signal Blocking MatriX}

From (10), we note that the construction of the required 2-D signal blocking matrix $\boldsymbol{B}$ must satisfy the condition of $\boldsymbol{C}^{H} \boldsymbol{B}=$ o. According to existing techniques like [7], the 2-D blocking matrix $\boldsymbol{B}=\left[b_{i j}\right]$ with size $M N \times(M N-K)$ is directly computed from the formula $\boldsymbol{C}^{H} \boldsymbol{B}=\mathbf{o}$. This leads to the need for complicated computations including the singular value decomposition (SVD) of $\boldsymbol{C}$, and hence, the required computational burden is $O\left(M^{3} N^{3}+K M^{2} N^{2}\right)$ [13]. In the following, we present a technique to efficiently construct the 2-D blocking matrix $B$ without the above complicated computations.

From (4), we note that the response vector of the $i$ th signal source $\boldsymbol{a}\left(u_{i}, v_{i}\right)=\boldsymbol{a}_{r}\left(v_{i}\right) \otimes \boldsymbol{a}_{c}\left(u_{i}\right)$ with $u_{i}$ given by $u_{i}=\pi \sin \left(\theta_{i}\right) \cos \left(\phi_{i}\right)$ and $v_{i}$ given by $\left.v_{i}=\pi \sin (\theta)_{i}\right) \sin \left(\phi_{i}\right)$. Let $Z_{a i}=\exp \left\{j u_{i}\right\}$ and $Z_{b i}=\exp \left\{j v_{i}\right\}$. Then, we have $\boldsymbol{a}_{c}\left(u_{i}\right)=\boldsymbol{a}_{c}\left(Z_{a i}\right)=\left[Z_{a i}^{(1-m 0)}, Z_{a i}^{(2-m 0)}, \ldots, Z_{a i}^{(M-m 0)}\right]^{T}$, $\boldsymbol{a}_{r}\left(v_{i}\right)=\boldsymbol{a}_{r}\left(Z_{b i}\right)=\left[Z_{b i}^{(1-n 0)}, Z_{b i}^{(2-n 0)}, \ldots, Z_{b i}^{(N-n 0)}\right]^{T}$, and $\boldsymbol{a}\left(u_{i}, v_{i}\right)=\boldsymbol{a}\left(Z_{a i}, Z_{b i}\right)=\boldsymbol{a}_{r}\left(Z_{b i}\right) \otimes \boldsymbol{a}_{c}\left(Z_{a i}\right)$. Accordingly, the constraint matrix can be expressed as $\boldsymbol{C}=\left[\boldsymbol{a}\left(Z_{a 1}, Z_{b 1}\right) \boldsymbol{a}\left(Z_{a 2}, Z_{b 2}\right) \ldots \boldsymbol{a}\left(Z_{a K}, Z_{b K}\right)\right]$.

The condition of $\boldsymbol{C}^{H} \boldsymbol{B}=\mathbf{0}$ indicates that $\boldsymbol{B}^{H} \boldsymbol{a}\left(Z_{a i}, Z_{b i}\right)=$ $0, i=1,2, \ldots, K$. We express the product $\boldsymbol{B}^{H} \boldsymbol{a}\left(Z_{a}, Z_{b}\right)$ as follows:

$$
\boldsymbol{B}^{H} \boldsymbol{a}\left(Z_{a}, Z_{b}\right)=\Phi\left(Z_{a}\right) \boldsymbol{a}_{x}\left(Z_{a}, Z_{b}\right)+\psi\left(Z_{b}\right) \boldsymbol{a}_{y}\left(Z_{a}, Z_{b}\right)
$$

where $\Phi\left(Z_{a}\right)$ and $\psi\left(Z_{b}\right)$ are given by

$$
\text { and } \begin{aligned}
\Phi\left(Z_{a}\right) & =\left(Z_{a}-Z_{a 1}\right)\left(Z_{a}-Z_{a 2}\right) \ldots\left(Z_{a}-Z_{a K}\right) \\
\psi\left(Z_{b}\right) & =\left(Z_{b}-Z_{b 1}\right)\left(Z_{b}-Z_{b 2}\right)\left(Z_{b}-Z_{b K}\right)
\end{aligned}
$$

respectively. $\Phi\left(Z_{a}\right)$ and $\psi\left(Z_{b}\right)$ are two algebraic expressions used to represent that the condition of $\boldsymbol{C}^{H} \boldsymbol{B}=\mathbf{o}$ must be satisfied at $Z_{a}=Z_{a i}$ and $Z_{a}=Z_{b i}, i=1,2, \ldots, K$. Moreover, $\boldsymbol{a}_{x}\left(Z_{a}, Z_{b}\right)$ and $\boldsymbol{a}_{y}\left(Z_{a}, Z_{b}\right)$ are two vectors used to stand for the remaining parts of $\boldsymbol{B}^{H} \boldsymbol{a}\left(Z_{a}, Z_{b}\right)$ after we extract the roots at $Z_{a}=Z_{a i}$ and $Z_{a}=Z_{b i}, i=1,2, \ldots, K$ and are given by

$$
\boldsymbol{a}_{x}\left(Z_{a}, Z_{b}\right)=\boldsymbol{a}_{r}\left(Z_{b}\right) \otimes \boldsymbol{a}_{c K}\left(Z_{a}\right)
$$

where $\boldsymbol{a}_{c K}\left(Z_{a}\right)=\left[Z_{a}^{(1-m 0)}, Z_{a}^{(2-m 0)}, \ldots, Z_{a}^{(M-K-m 0)}\right]^{T}$ and

$$
\boldsymbol{a}_{y}\left(Z_{a}, Z_{b}\right)=\boldsymbol{a}_{r K}\left(Z_{b}\right) \otimes \boldsymbol{a}_{c}\left(Z_{a}\right)
$$

with $\boldsymbol{a}_{r K}\left(Z_{b}\right)=\left[Z_{b}^{(1-n 0)}, Z_{b}^{(2-n 0)}, \ldots, Z_{b}^{(N-K-n 0)}\right]^{T}$, respectively. In order to make the equality in the dimension for the vectors shown in (11), $(M-N) K$ zero entries must be appended to the vector $\boldsymbol{a}_{y}\left(Z_{a}, Z_{b}\right)$ since $(M-K) N>M(N-K)$ if $M>N$. Hence, both $\boldsymbol{a}_{x}\left(Z_{a}, Z_{b}\right)$ and $\boldsymbol{a}_{y}\left(Z_{a}, Z_{b}\right)$ have size $(M-K) N \times 1$. In contrast, $(N-M) K$ zero entries must be appended to the vector $\boldsymbol{a}_{x}\left(Z_{a}, Z_{b}\right)$ since $(M-K) N<M(N-K)$ if $M<N$. Thus, both $\boldsymbol{a}_{x}\left(Z_{a}, Z_{b}\right)$ and $\boldsymbol{a}_{y}\left(Z_{a}, Z_{b}\right)$ have size $(N-K) M \times 1$. Since $\boldsymbol{a}\left(Z_{a}, Z_{b}\right)=\boldsymbol{a}_{r}\left(Z_{b}\right) \otimes \boldsymbol{a}_{c}\left(Z_{a}\right)=$ $\left[Z_{a}^{(1-m 0)} Z_{b}^{(1-n 0)}, Z_{a}^{(2-m 0)} Z_{b}^{(1-n 0)} Z_{a}^{(3-m 0)} Z_{b}^{(1-n 0)}, \ldots\right.$, $Z_{a}^{(M-m 0)} Z_{b}^{(1-n 0)}, Z_{a}^{(1-m 0)} Z_{b}^{(2-n 0)}, \ldots, Z_{a}^{(M-m 0)} Z_{b}^{(2-n 0)}$, $\left.Z_{a}^{(1-m 0)} Z_{b}^{(3-n 0)}, \ldots, Z_{a}^{(M-m 0)} Z_{b}^{(N-n 0)}\right]^{T}$, we have the $i$ th entry of the vector $\boldsymbol{r}=\boldsymbol{B}^{H} \boldsymbol{a}\left(Z_{a}, Z_{b}\right)=\left[r_{i}\right]^{T}$, which is given by

$$
\begin{aligned}
& r_{i}=b_{i 1} Z_{a}^{(1-m 0)} Z_{b}^{(1-n 0)}+b_{i 2} Z_{a}^{(2-m 0)} Z_{b}^{(1-n 0)} \\
& +b_{i 3} Z_{a}^{(3-m 0)} Z_{b}^{(1-n 0)}+\cdots+b_{i M N} Z_{a}^{(M-m 0)} Z_{b}^{(N-n 0)}
\end{aligned}
$$


for $i=1,2, \ldots,(M-K) N$ if $M>N$ and $i=1,2, \ldots,(N-$ $K) M$ if $N>M$. Based on the results of (11), (12), and (15), we can compare each entry corresponding to the both sides of (11) to find the $b_{i j}, i=1,2, \ldots M N$, and $j=1,2, \ldots,(M-K) N$ if $M>N$ or $j=1,2, \ldots,(N-K) M$ if $N>M$, which required to construct the 2-D signal blocking matrix $B$ with size $M N \times(M-K) N$ if $M>N$ and $M N \times(N-K) M$ if $N>M$. For illustration, we present the formulas to compute the entries $b_{i j}$ of $B$ as follows. For $M=N$ and $K=1$, (12) becomes $\Phi\left(Z_{a}\right)=\left(Z_{a}-Z_{a 1}\right)$, and $\Psi\left(Z_{b}\right)=\left(Z_{b}-Z_{b 1}\right)$. According to (15), we can easily find $b_{i j}$ equal to

$$
b_{i j}= \begin{cases}-\left(Z_{a 1}+Z_{b 1}\right), & \text { for } i=j \\ 1, & \text { for } i=j+1 \\ 1, & \text { for } i=j+M \\ 0, & \text { otherwise }\end{cases}
$$

for $j=1,2, \ldots, M-1$.

$$
b_{i j}= \begin{cases}-Z_{b 1}, & \text { for } i=j \\ -Z_{a 1}, & \text { for } i=j+1 \\ 1, & \text { for } i=j+2 \\ 1, & \text { for } i=j+M \\ 0, & \text { otherwise }\end{cases}
$$

for $j=M, M+1, \ldots, 2 M-2$.

$$
b_{i j}= \begin{cases}-Z_{b 1}, & \text { for } i=j \\ -Z_{a 1}, & \text { for } i=j+2 \\ 1, & \text { for } i=j+3 \\ 1, & \text { for } i=j+M \\ 0, & \text { otherwise }\end{cases}
$$

for $j=2 M-1,2 M, \ldots, 3 M-3$.

$$
b_{i j}= \begin{cases}-Z_{b 1}, & \text { for } i=j \\ -Z_{a 1}, & \text { for } i=j+(N-1) \\ 2, & \text { for } i=j+M \\ 0, & \text { otherwise }\end{cases}
$$

for $j=(M-1)(N-1)+1, \ldots, N(M-1)$.

Following the similar procedure, we can also find the entries $b_{i j}$ of the resulting 2-D signal blocking matrix $B^{H}$ for the case of $M=N$ and $K=2$, which is given as (20)-(22), shown at the bottom of the page. As far as the general case of $M \neq N$, i.e., a 2-D rectangular array, we present the resulting $B$ in the case of $M=5$ and $N=4$ for illustration as follows. First, the response vector becomes $\boldsymbol{a}\left(Z_{a}, Z_{b}\right)=\boldsymbol{a}_{r}\left(Z_{b}\right) \otimes \boldsymbol{a}_{c}\left(Z_{a}\right)$ with $\boldsymbol{a}_{c}\left(Z_{a}\right)=\left[Z_{a}^{(1-m 0)}, Z_{a}^{(2-m 0)}, \ldots, Z_{a}^{(5-m 0)}\right]^{T}$ and $\boldsymbol{a}_{r}\left(Z_{b}\right)$ $=\left[Z_{b}^{(1-n 0)}, Z_{b}^{(2-n 0)}, \ldots, Z_{b}^{(4-n 0)}\right]^{T}$. Therefore, $\boldsymbol{a}\left(Z_{a}, Z_{b}\right)$ $=\boldsymbol{a}_{r}\left(Z_{b}\right) \otimes \boldsymbol{a}_{c}\left(Z_{a}\right)=\left[Z_{a}^{(1-m 0)} Z_{b}^{(1-n 0)}, Z_{a}^{(2-m 0)} Z_{b}^{(1-n 0)}\right.$ $Z_{a}^{(3-m 0)} Z_{b}^{(1-n 0)}, \quad \ldots, Z_{a}^{(5-m 0)} Z_{b}^{(1-n 0)}, Z_{a}^{(1-m 0)} Z_{b}^{(2-n 0)}$, $\ldots, Z_{a}^{(5-m 0)} Z_{b}^{(2-n 0)}, Z_{a}^{(1-m 0)} Z_{b}^{(3-n 0)}, \ldots, Z_{a}^{(5-m 0)} Z_{b}^{(4-n 0)}$ ]$^{T}$. For $K=1$, (12) becomes $\Phi\left(Z_{a}\right)=\left(Z_{a}-Z_{a 1}\right)$ and $\psi\left(Z_{b}\right)=\left(Z_{b}-Z_{b 1}\right)$. According to (15), we can easily find $b_{i j}$ equal to

$$
\begin{aligned}
& b_{i j}= \begin{cases}-\left(Z_{a 1}+Z_{b 1}\right), & \text { for } i=j \\
1, & \text { for } i=j+1 \\
1, & \text { for } i=j+5 \\
0, & \text { otherwise }\end{cases} \\
& \text { for } j=1,2, \ldots, 4 \text {. } \\
& b_{i j}= \begin{cases}-Z_{b 1}, & \text { for } i=j \\
-Z_{a 1}, & \text { for } i=j+1 \\
1, & \text { for } i=j+2 \\
1, & \text { for } i=j+5 \\
0, & \text { otherwise }\end{cases} \\
& \text { for } j=5, \ldots, 8 \text {. } \\
& b_{i j}= \begin{cases}-Z_{b 1}, & \text { for } i=j \\
-Z_{a 1}, & \text { for } i=j+2 \\
1, & \text { for } i=j+3 \\
1, & \text { for } i=j+5 \\
0, & \text { otherwise }\end{cases} \\
& \text { for } j=9, \ldots, 12 \text {. }
\end{aligned}
$$

for $j=M-1, M, \ldots, 2 M-4$.

$$
b_{i j}= \begin{cases}Z_{b 1} Z_{b 2}, & \text { for } i=j \\ Z_{a 1} Z_{a 2}, & \text { for } i=j+2(N-2) \\ -\left(Z_{a 1}+Z_{a 2}\right), & \text { for } i=j+2(N-2)+1 \\ -\left(Z_{b 1}+Z_{b 2}\right), & \text { for } i=j+M \\ 2, & \text { for } i=j+2 M \\ 0, & \text { otherwise }\end{cases}
$$

for $j=(M-2)(N-1)+1, \ldots,(M-2) N$. 
To find the entries $b_{i j}, j=13, \ldots, 16, i=1,2, \ldots, 20$, we have to utilize the formula given by (11) and compare each entry corresponding to the both sides of (11). The resulting 2-D signal blocking matrix $\boldsymbol{B}^{H}$ for the case of $K=1$ is given as in (26), shown at the bottom of the page. Following a similar procedure, we can also find the resulting 2-D signal blocking matrix $\boldsymbol{B}^{H}$ for the case of $K=2$ given as in (27), shown at the bottom of the page.

From the constructed 2-D signal blocking matrix $B$, we observe that it has size of $M N \times(\max \{M, N\}-K) \min \{M, N\}$, where $\max \{M, N\}$ denotes the maximum of $M$ and $N$, and $\min \{M, N\}$ the minimum of $M$ and $N$. Therefore, the proposed 2-D signal blocking matrix $\boldsymbol{B}$ requires significantly fewer entries as compared with the one proposed by Salem et al. [7] with size $M N \times(M N-K)$. This reveals that the proposed GSC-based 2-D array beamformer can provide satisfactory performance by requiring only $(\max \{M, N\}-K) \min \{M, N\}$ instead of $(M N-K)$ adaptive weights.

Finally, we evaluate the computational complexity in terms of complex multiplications $(\mathrm{CM})$ required for constructing the 2-D signal blocking matrix $\boldsymbol{B}$ using the proposed method. Here, we describe the number of the CMs required for the construction of the proposed 2-D signal blocking matrix $\boldsymbol{B}$ for $K$ desired signals as follows:
1) For $K=1$, we follow the formulas of (12), which become $\Phi\left(Z_{a}\right)=\left(Z_{a}-Z_{a 1}\right)$ and $\Phi\left(Z_{b}\right)=\left(Z_{b}-Z_{b 1}\right)$, respectively. Hence, computing the coefficients by (15) requires no $\mathrm{CM}$ at all. 2) For $K=2$, the formulas of (12) become $\Phi\left(Z_{a}\right)=\left(Z_{a}-\right.$ $\left.Z_{a 1}\right)\left(Z_{a}-Z_{a 2}\right)$ and $\Psi\left(Z_{b}\right)=\left(Z_{b}-Z_{b 1}\right)\left(Z_{b}-Z_{b 2}\right)$, respectively. Computing the coefficients by (15) requires the CM for obtaining $Z_{a 1} Z_{a 2}$ and $Z_{b 1} Z_{b 2}$, i.e., the number of $\mathrm{CM}$ required is $2=2 \times_{2} C_{2}=2 \sum_{k=2}^{K}{ }_{K} C_{k}$. 3) For $K=3$, the formulas of (12) become $\Phi\left(Z_{a}\right)=\left(Z_{a}-Z_{a 1}\right)\left(Z_{a}-Z_{a 2}\right)\left(Z_{a}-Z_{a 3}\right)$ and $\Psi\left(Z_{b}\right)=\left(Z_{b}-Z_{b 1}\right)\left(Z_{b}-Z_{b 2}\right)\left(Z_{b}-Z_{b 3}\right)$, respectively. Computing the coefficients by (15) requires the CM for obtaining $Z_{a 1} Z_{a 2}, Z_{a 1} Z_{a 3}, Z_{a 2} Z_{a 3}, Z_{a 1} Z_{a 2} Z_{a 3}$, and $Z_{b 1} Z_{b 2}, Z_{b 1} Z_{b 3}$, $Z_{b 2} Z_{b 3}, Z_{b 1} Z_{b 2} Z_{b 3}$, i.e., the number of $\mathrm{CM}$ required is $8=$ $2 \times\left[{ }_{3} C_{2}+{ }_{3} C_{3}\right]=2 \times[3+1]=2 \sum_{k=2}^{K}{ }_{K} C_{k}$. Following the similar procedure described above, we can derive the general formula for calculating the number $T_{\mathrm{CM}}$ of $\mathrm{CM}$ required for any value of $K$ as follows:

$$
T_{\mathrm{CM}}=2 \sum_{k=2}^{K}{ }_{K} C_{k}
$$

where ${ }_{K} C_{k}=K ! /[(K-k) ! k !]$ represents the number of $k$-element combinations of $K$ objects. Clearly, $T_{\mathrm{CM}}$ is significantly less than that required by using the conventional method [7].

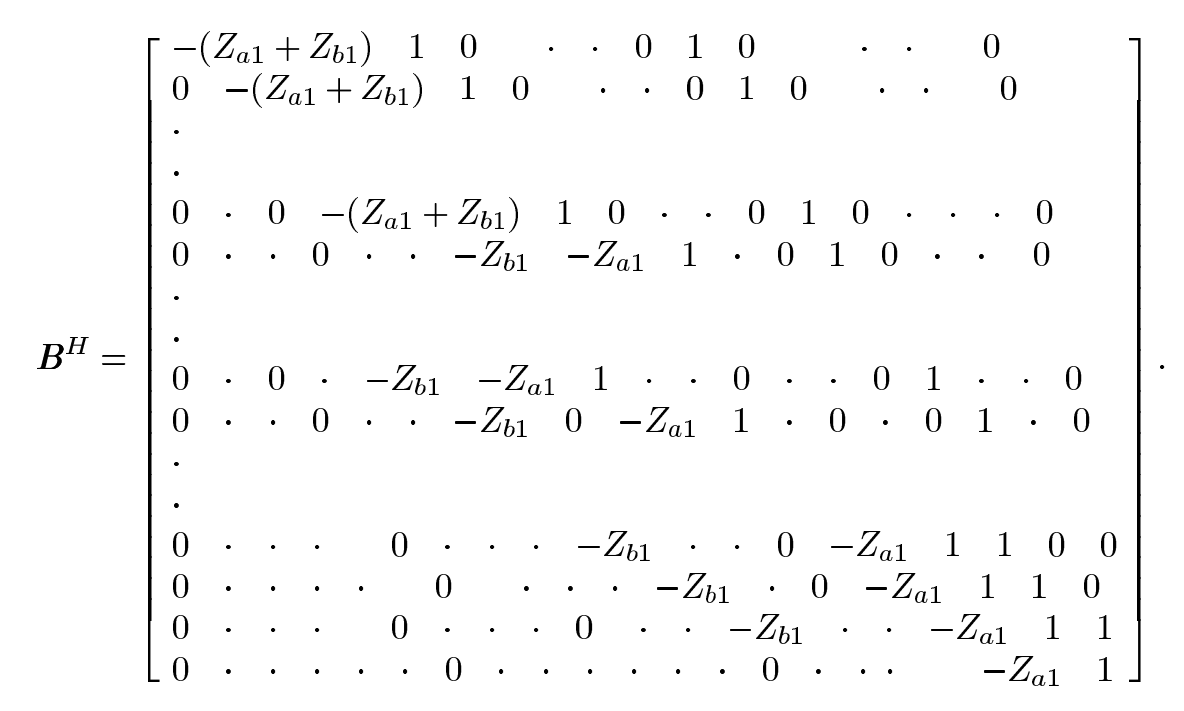

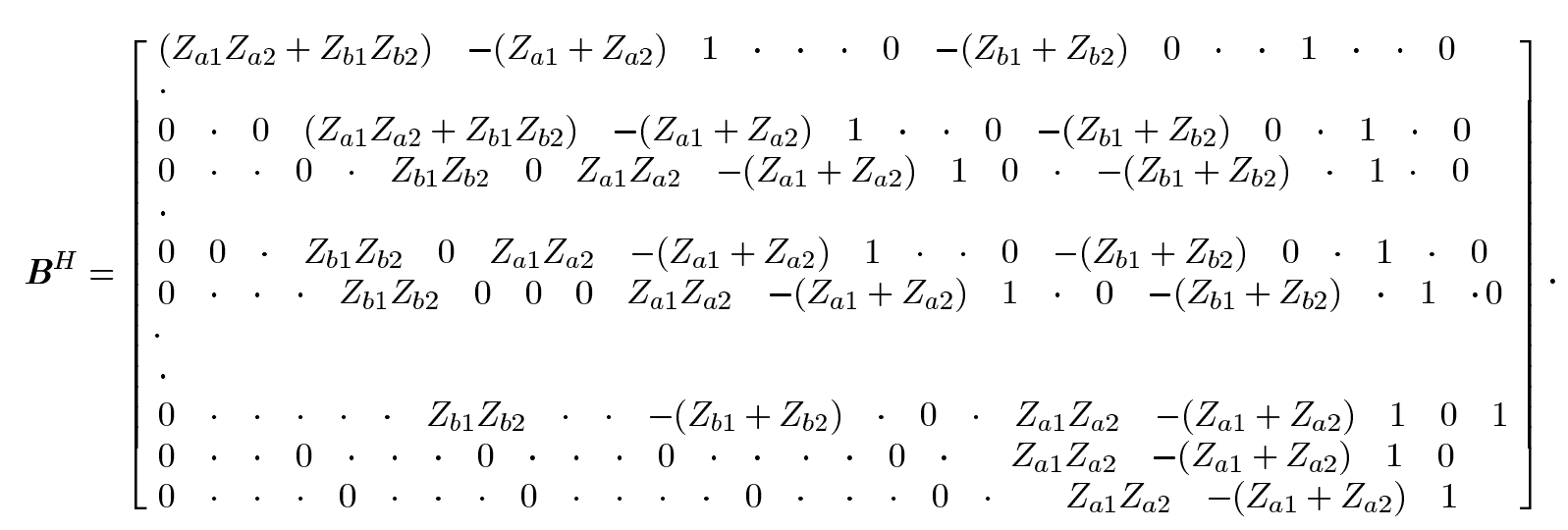




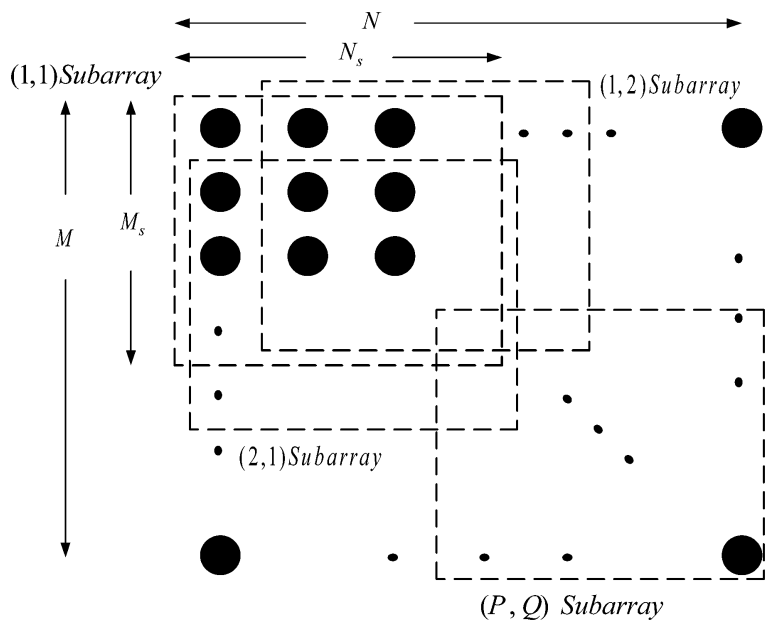

Fig. 3. Subarray grouping for a 2-D uniform planar array.

\section{Two-Dimensional Weighted Spatial SMOOTHING SCHEME}

In the presence of coherence between the desired signals and interferers, the performance of a 2-D array beamformer deteriorates due to the signal cancellation phenomenon. According to the approach proposed by [14], we first briefly describe the 2-D spatial smoothing (2-D SS) scheme for a 2-D uniform planar array (UPA). For simplicity, we use a simple example for demonstration. Let the $M \times N$ 2-D UPA be grouped into $P \times Q$ overlapping subarrays of size $M_{s} \times N_{s}$, as shown in Fig. 3 . Assume that $\boldsymbol{x}_{(i, j)}(t)$ represents the input vector of the $(i, j)$ th subarray, which consists of the $i$ th to the $(M-P+i)$ th rows and the $j$ th to the $(N-Q+j)$ th columns of the 2-D UPA. The autocorrelation matrix of the $(i, j)$ th subarray is then given by $\boldsymbol{R}_{x}^{(i, j)}=E\left\{\boldsymbol{x}_{(i, j)}(t) \boldsymbol{x}_{(i, j)}^{H}(t)\right\}$. Following the 2-D SS scheme of [14], we obtain the 2-D spatially smoothed autocorrelation matrix by taking $\boldsymbol{R}_{x}^{a}=(1 / p \times Q) \sum_{i=1}^{P} \sum_{j=1}^{Q} \boldsymbol{R}_{x}^{(i, j)}$. In order to successfully decorrelate $D$ coherent signals, the condition $P \times Q \geqq D$ must be held. Then, $\boldsymbol{R}_{x}^{a}$ is used instead of $\boldsymbol{R}_{x}$, which is shown by (5) to perform 2-D adaptive beamforming. However, similar to the 1-D case, the effectiveness of the conventional 2-D SS is limited because the effective aperture size is reduced due to $M_{s}<M$ and $N_{s}<N$. Moreover, the 2-D spatially smoothed autocorrelation matrix $\boldsymbol{R}_{x}^{a}$ cannot possess the block Toeplitz property from the simple averaging on $\boldsymbol{R}_{x}^{(i, j)}$.

To tackle the drawbacks of using conventional 1-D spatial smoothing scheme of [15], Takao et al. [16] presented a so-called weighted spatial smoothing (WSS) scheme, which adapts the variable weights instead of uniform weighting to obtain a 1-D spatially smoothed autocorrelation matrix. The variable weights are found through a Toeplitzization algorithm that makes the resultant spatially smoothed matrix Toeplitz. Recently, this technique of [16] was extended by Indukumar $e t$ $a l$. [17] to solve the coherent problem when dealing with broadband signals. In the literature, there are practically no papers considering an appropriate WSS scheme that deals with the case of using a 2-D planar array. In the following, we introduce a 2-D WSS scheme to tackle the coherence between signal
TABLE I

CONCATENATION OF EQUATIONS AND RELATIONS FOR THE 2-D WSS SCHEME

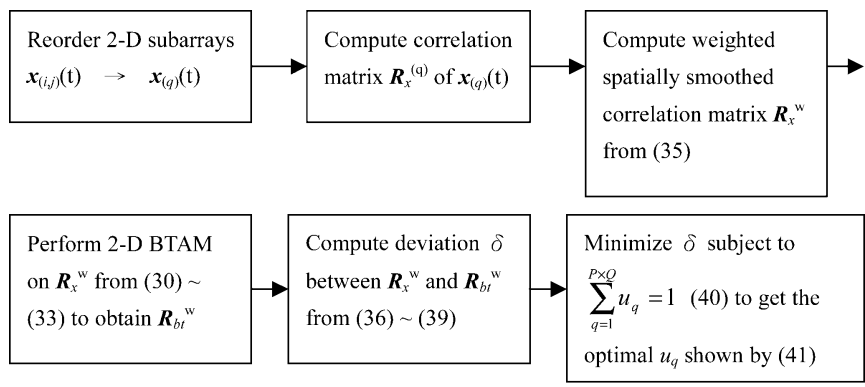

sources and to ensure that the obtained spatially smoothed autocorrelation matrix is block Toeplitz.

Let the sampled version of the 2-D autocorrelation matrix $\boldsymbol{R}_{x}$ be $\boldsymbol{R}_{x}(L)$, which is obtained by taking $L$ data snapshots. We express $\boldsymbol{R}_{x}(L)$ as follows:

$$
\boldsymbol{R}_{x}(L)=\left[\begin{array}{cccc}
\boldsymbol{R}_{11} & \boldsymbol{R}_{12} & \ldots & \boldsymbol{R}_{1 N} \\
\boldsymbol{R}_{21} & \boldsymbol{R}_{22} & \ldots & \boldsymbol{R}_{2 N} \\
\vdots & \vdots & \vdots & \vdots \\
\boldsymbol{R}_{N 1} & \boldsymbol{R}_{N 2} & \ldots & \boldsymbol{R}_{N N}
\end{array}\right]
$$

where $\boldsymbol{R}_{i j}$ denotes the $M \times M$ cross-correlation matrix of the data vectors received by the $i$ th and $j$ th column subarrays, respectively. Then, a 2-D block-Toeplitz approximation method (2-D BTAM) is presented to construct an $M N \times M N$ block Toeplitz matrix as follows:

$$
\begin{aligned}
\boldsymbol{R}_{b t}(L) & =\boldsymbol{B} T\left[\boldsymbol{R}_{x}(L)\right] \\
& =\left[\begin{array}{cccc}
\boldsymbol{R}_{0} & \boldsymbol{R}_{-1} & \cdots & \boldsymbol{R}_{(1-N)} \\
\boldsymbol{R}_{-1}^{H} & \boldsymbol{R}_{0} & \cdots & \boldsymbol{R}_{(2-N)} \\
\vdots & \vdots & \vdots & \vdots \\
\boldsymbol{R}_{(1-N)}^{H} & \boldsymbol{R}_{(2-N)}^{H} & \cdots & \boldsymbol{R}_{0}
\end{array}\right]
\end{aligned}
$$

where

$$
\mathbf{R}_{-n}=\frac{1}{N-n} \sum_{i=1}^{N-n} \boldsymbol{T}\left[\boldsymbol{R}_{i(i+n)}\right], \quad n=0,1,2, \ldots, N-1
$$

are all Toeplitz matrices with size $M \times M$, and $\boldsymbol{T}[\boldsymbol{R}]$ denotes the Toeplitzization operation performed on matrix $\boldsymbol{R}$ and is given by

$$
T[R]=\left[r_{i j}\right]=[r(i-j)]
$$

where

$$
\begin{aligned}
& r(-m)=\frac{1}{M-m} \sum_{i=1}^{M-m} r_{i(i+m)}, \quad 0 \leqq m<M \\
& r(-m)=r(m)^{*}
\end{aligned}
$$

and $r_{i(i+m)}$ represents the $(i, i+m)$ th entry of $\boldsymbol{R}$. The subscript "bt" in (30) indicates that the obtained matrix $R_{b t}(L)$ possesses the block Toeplitz property. We note that $\boldsymbol{R}_{t}=\boldsymbol{T}[\boldsymbol{R}]$ is the solution of the following minimization problem:

$$
\text { Minimize }\left\|\boldsymbol{R}_{t}-\boldsymbol{R}\right\|, \quad \boldsymbol{R}_{t} \in S_{t}
$$


where $S_{t}$ represents the set of Toeplitz matrices with size $M \times$ $M$, and $\|\boldsymbol{A}\|$ denotes the Frobenius norm of the matrix $\boldsymbol{A}[18]$ Moreover, (30) reveals that the constructed matrix $\boldsymbol{R}_{b t}(L)$ is also Hermitian.

Based on the above 2-D SS and 2-D BTAM schemes, we introduce a 2-D WSS as follows. First, we reorder the 2-D subarrays. Let the $(i, j)$ th 2 -D subarray be denoted by the $q$ th $2-\mathrm{D}$ subarray according to the relationship of $q=i+(j-1) \times P$. Then, we perform the 2-D SS with imposed weights on the 2-D UPA to obtain the 2-D spatially smoothed autocorrelation matrix $\boldsymbol{R}_{x}^{w}$ as follows:

$$
\boldsymbol{R}_{x}^{w}=\sum_{q=1}^{P \times Q} u_{q} \boldsymbol{R}_{x}^{(q)}
$$

where $u_{q}$ are the real weights to be chosen subject to $\sum_{q=1}^{P \times Q} u_{q}=1$ for taking the average from the autocorrelation matrix $\boldsymbol{R}_{x}^{(q)}$ of the $q$ th 2-D subarray. Then, the above 2-D BTAM is performed on $\boldsymbol{R}_{x}^{w}$ to produce $\boldsymbol{R}_{b t}^{w}=\boldsymbol{B} \boldsymbol{T}\left[\boldsymbol{R}_{x}^{w}\right]$. We define a parameter $\delta$ to represent the measure of deviation of $\boldsymbol{R}_{x}^{w}$ from $\boldsymbol{R}_{b t}^{w}$ as follows:

$$
\delta=\sum_{h=0}^{M_{s} N_{s}-2} \sum_{g=1}^{M_{s} N_{s}-h}\left|R_{x}^{w}(g+h, g)-R_{b t}^{w}(g+h, g)\right|^{2}
$$

where $R_{x}^{w}(g+h, g)$ and $R_{b t}^{w}(g+h, g)$ denote the $(g+h, g)$ th elements of $\boldsymbol{R}_{x}^{w}$ and $\boldsymbol{R}_{b t}^{w}$, respectively. In matrix form, we rewrite (36) as follows:

$$
\delta=\boldsymbol{u}^{T} \boldsymbol{R}_{e e} \boldsymbol{u}
$$

where $\boldsymbol{u}=\left[u_{1}, u_{2}, \ldots, u_{P Q}\right]^{T}$ denotes the weighting vector and $\boldsymbol{R}_{e e}$ is given by

$$
\boldsymbol{R}_{e e}=\sum_{h=0}^{M_{s} N_{s}-2} \sum_{g=1}^{M_{s} N_{s}-h} \operatorname{Re}\left\{\boldsymbol{z}_{g+h, g} \boldsymbol{z}_{g+h, g}{ }^{\mathrm{H}}\right\}
$$

where the $P Q \times 1$ vector $z_{g+h, g}$ has the $q$ th entry $z_{g+h, g}(q)$ given by

$$
\begin{aligned}
z_{g+h, g}(q) & =R_{x}^{(q)}(g+h, g)-R_{b t}^{(q)}(g+h, g), \\
q & =1,2, \ldots, P \times Q
\end{aligned}
$$

where $R_{x}^{(q)}(g+h, g)$ and $R_{b t}^{(q)}(g+h, g)$ represent the $(g+$ $h, g)$ th elements of $\boldsymbol{R}_{x}^{(q)}$ and $\boldsymbol{R}_{b t}^{(q)}=\boldsymbol{B} \boldsymbol{T}\left[\boldsymbol{R}_{x}^{(q)}\right]$, respectively. The optimal weights can be found by performing the following optimization problem:

$$
\begin{array}{ll}
\text { Minimize } & \boldsymbol{u}^{T} \boldsymbol{R}_{e e} \boldsymbol{u} \\
\text { Subject to } & \boldsymbol{u}^{T} \mathbf{1}=1
\end{array}
$$

where the $P Q \times 1$ vector $1=[1,1, \ldots, 1]^{T}$. The optimal solution for (40) is given by

$$
\boldsymbol{u}_{o}=\boldsymbol{R}_{e e}^{-1} \mathbf{1}\left(\mathbf{1}^{T} \boldsymbol{R}_{e e}^{-1} \mathbf{1}\right)^{-1}
$$

Table I lists the concatenation of the equations and the relations for the 2-D WSS scheme. Substituting $\boldsymbol{u}_{o}$ into (35) yields a spatially smoothed autocorrelation matrix $R_{x}^{w}$ that is very close to a

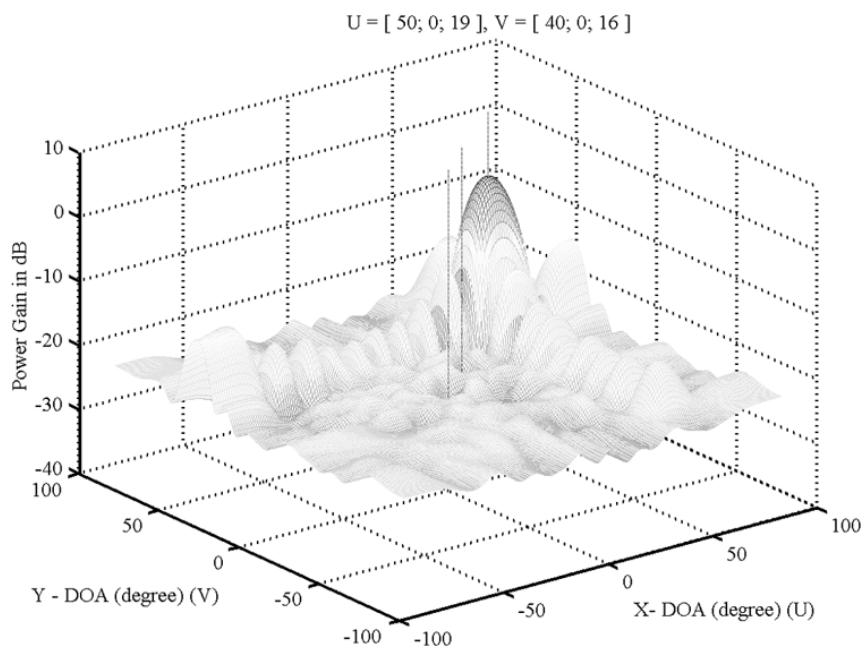

(a)

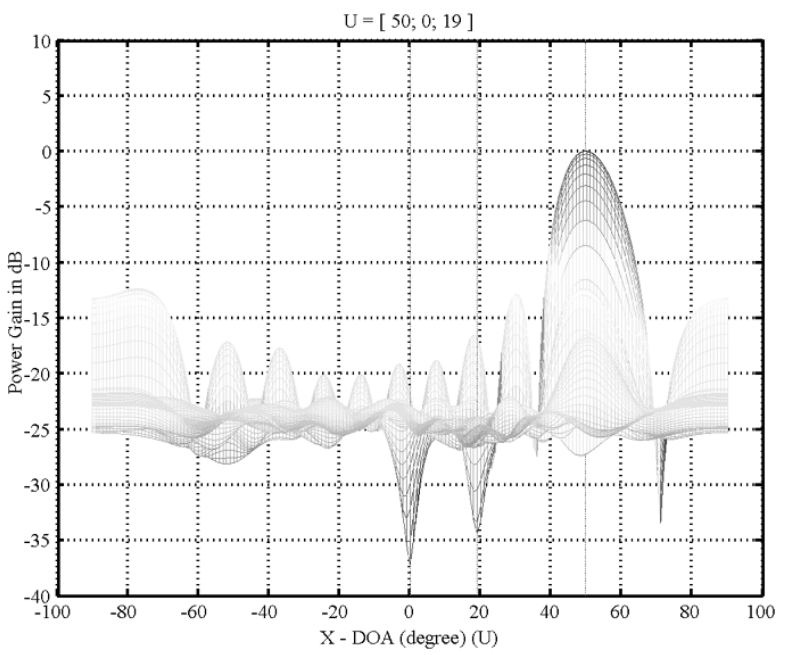

(b)

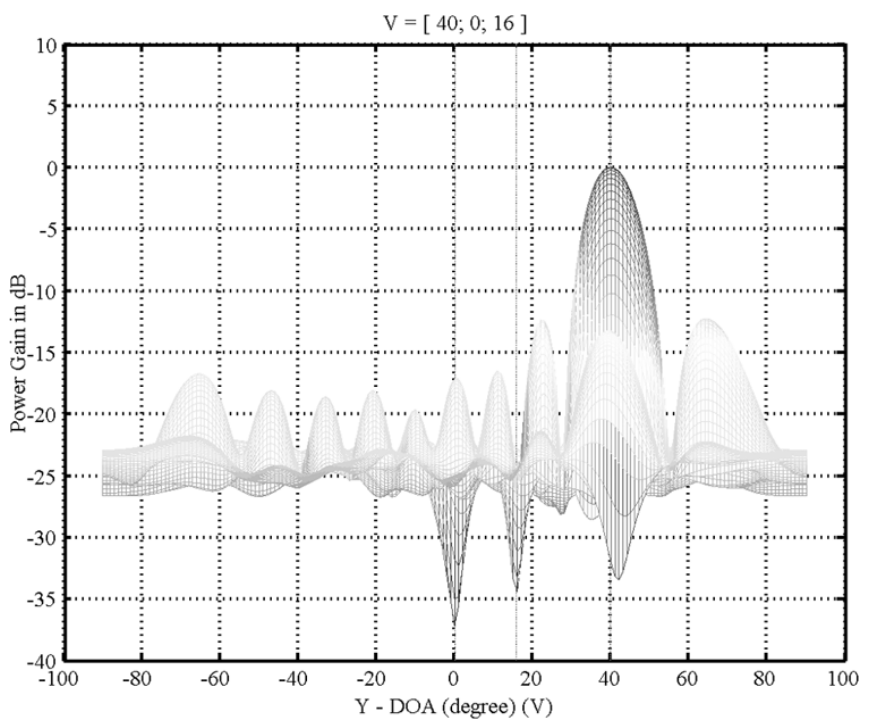

(c)

Fig. 4. (a) Two-dimensional array beam pattern without coherence for Example 1. (b) Beam pattern in the $U$ direction without coherence for Example 1. (c) Beam pattern in the $V$ direction without coherence for Example 1.

block Toeplitz structure. Due to spatial smoothing, the effective 2-D aperture size becomes $M_{s} \times N_{s}$. We construct a 2-D signal 


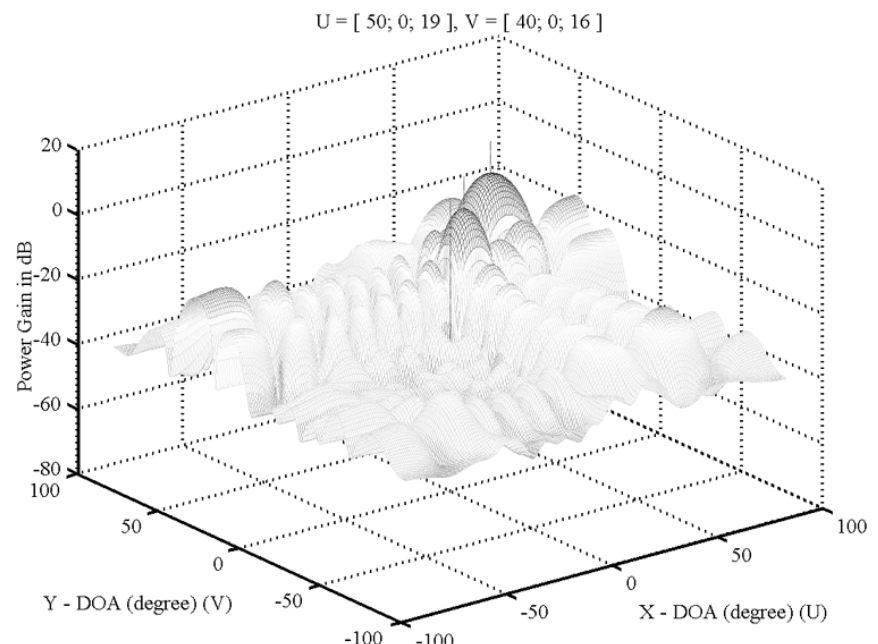

(a)

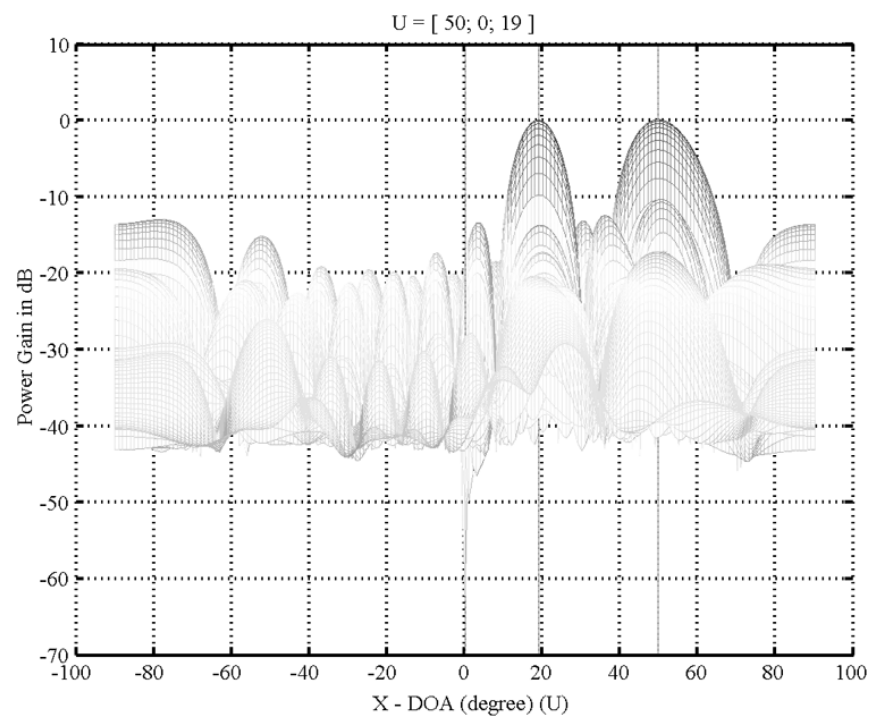

(ab)

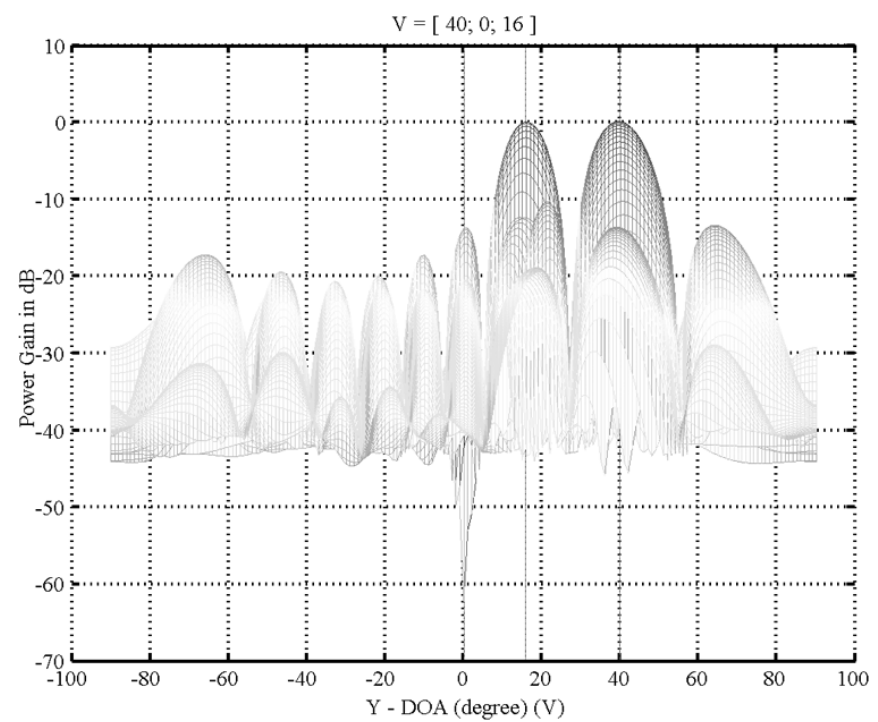

(c)

Fig. 5. (a) Two-dimensional array beam pattern with coherence for Example 1. (b) Beam pattern in the $U$ direction with coherence for Example 1. (c) Beam pattern in the $V$ direction with coherence for Example 1.

blocking matrix $\boldsymbol{B}$ using the technique presented in Section III with 2-D array size $M_{s} \times N_{s}$ instead of $M \times N$, and we com-

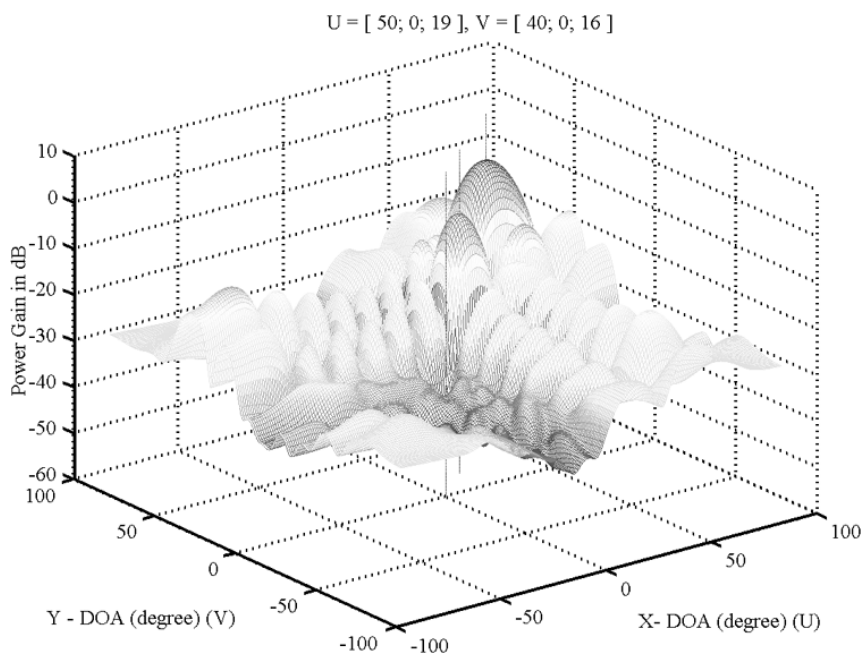

(a)

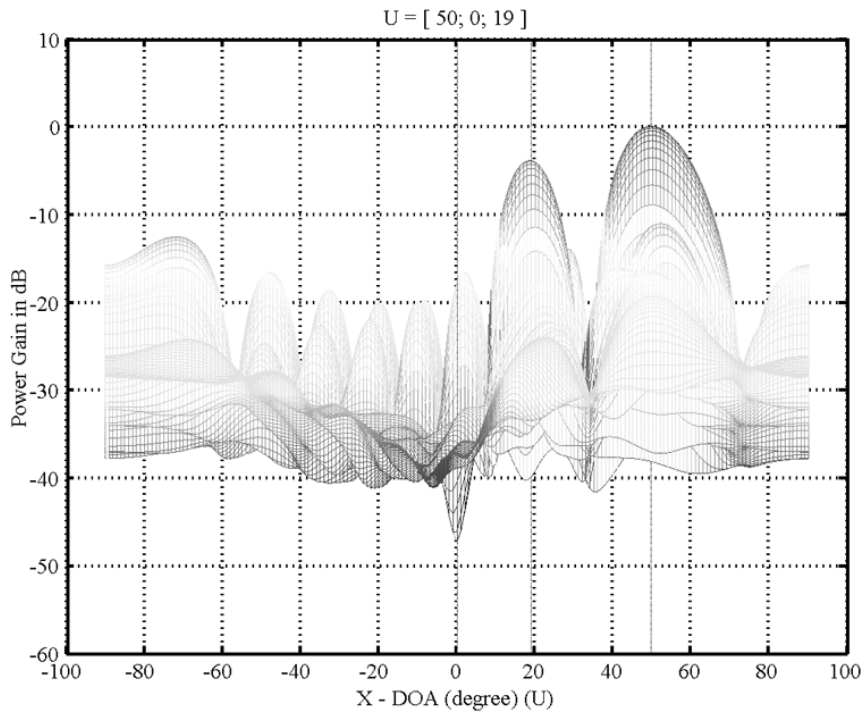

(b)

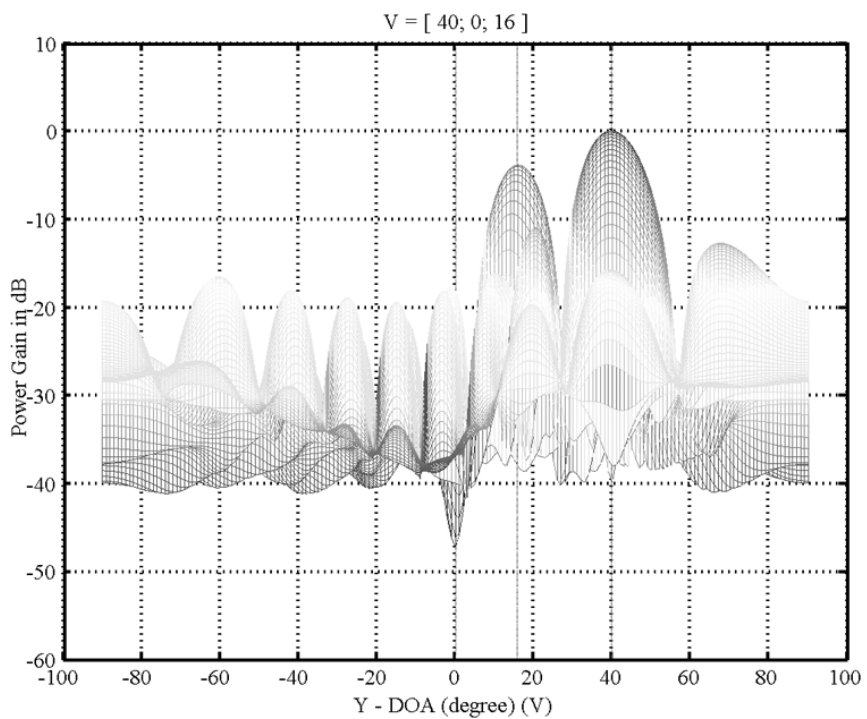

(c)

Fig. 6. (a) Two-dimensional array beam pattern after using 2-D SS for Example 1. (b) Beam pattern in the $U$ direction after using 2-D SS for Example 1. (c) Beam pattern in the $V$ direction after using 2-D SS for Example 1.

pute the overall weight vector from (10) based on the obtained $\boldsymbol{R}_{x}^{w}$ with size $M_{s} N_{s} \times M_{s} N_{s}$. 


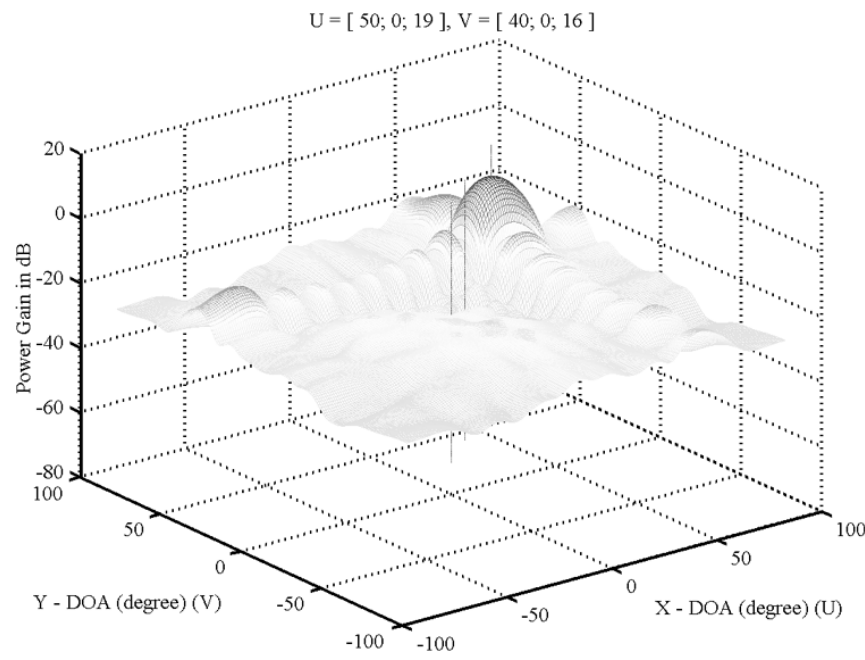

(a)

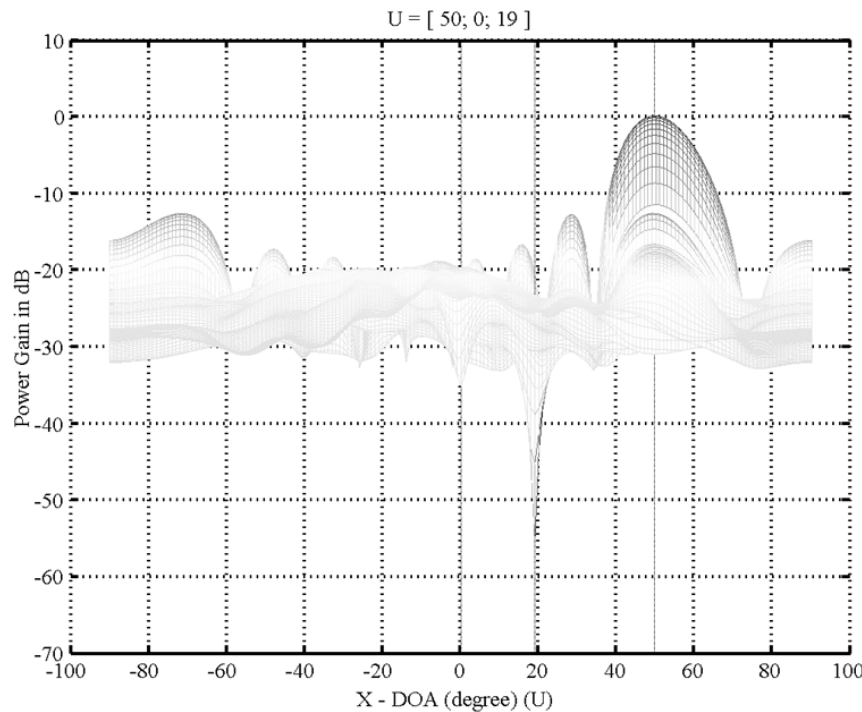

(b)

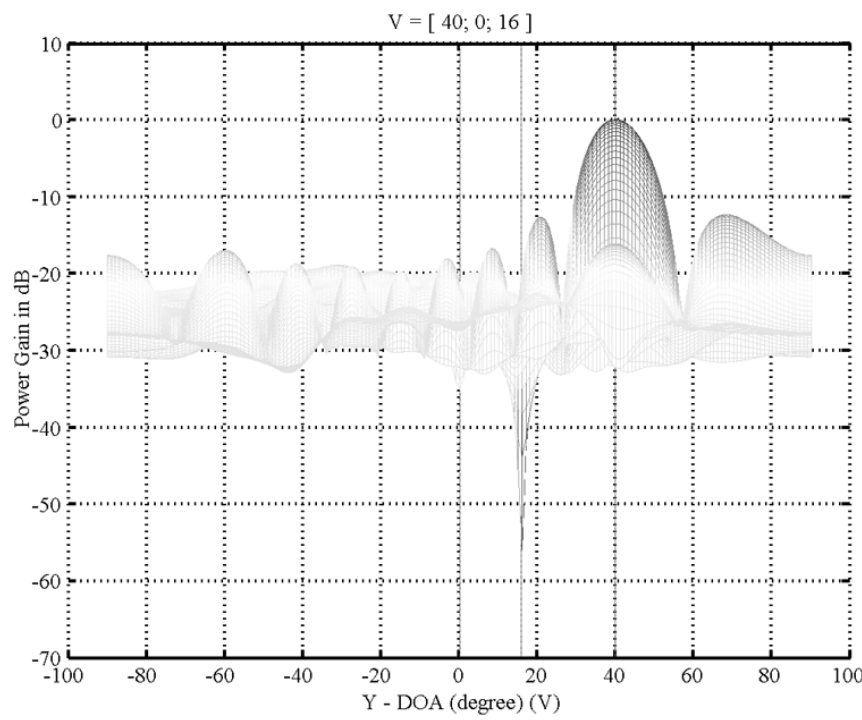

(c)

Fig. 7. (a) Two-dimensional array beam pattern after using 2-D WSS for Example 1. (b) Beam pattern in the $U$ direction after using 2-D WSS for Example 1. (c) Beam pattern in the $V$ direction after using 2-D WSS for Example 1.

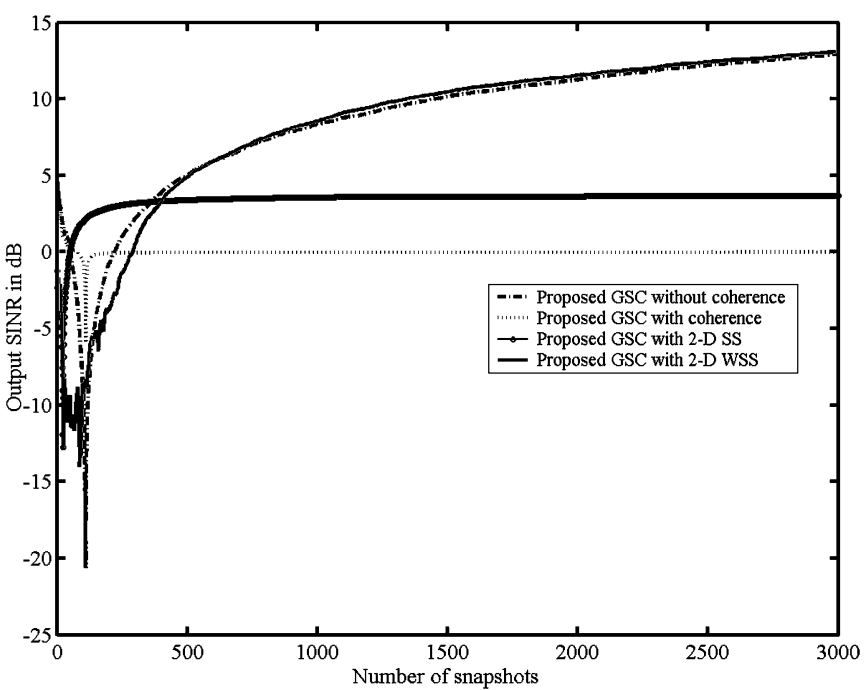

Fig. 8. Output SINR versus number of snapshots for Example 1.

TABLE II

OUTPUT SINRs (IN DECIBELS) OF USING DIFFERENT METHODS FOR EXAMPLE 1

\begin{tabular}{l|c|c|c|c}
\hline & $\begin{array}{l}\text { Without } \\
\text { Coherence }\end{array}$ & With Coherence & Using 2-D SS & $\begin{array}{l}\text { Using 2-D } \\
\text { WSS }\end{array}$ \\
\hline $\begin{array}{l}\text { Proposed } \\
\text { Method }\end{array}$ & 19.0792 & 0.000738398 & 3.73926 & 18.6704 \\
\hline $\begin{array}{l}\text { Traditional } \\
\text { 2-D GSC } \\
\text { Method [7] }\end{array}$ & 18.9632 & $1.08355 \times 10^{-5}$ & 3.73781 & 18.5729 \\
\hline $\begin{array}{l}\text { Conventional } \\
\text { LCMV } \\
\text { Method [11] }\end{array}$ & 18.9632 & $1.08355 \times 10^{-5}$ & 3.73781 & 18.5729 \\
\hline
\end{tabular}

\section{COMPUTER Simulation EXAMPleS}

In this section, several simulation examples performed on a Pentium IV PC using the MATLAB programming language are presented for illustration, confirming the theoretical works. The array used for all simulations is a 2-D UPA with the interelement spacing equal to $\lambda / 2$, where $\lambda$ is the smallest wavelength of the desired signals. All signals used for simulations are binary phase shift keying (BPSK) narrowband signals with cosine waveform shape and carrier frequency equal to $1 \mathrm{~Hz}$. The array phase center is set to the $(6,6)$ th sensor. All simulation results presented are obtained by averaging 30 independent runs with independent noise samples for each run. The received noise is spatially white noise with variance equal to 1 and zero mean. Moreover, in dealing with the case of coherent signal sources, the simulation results using conventional 2-D SS are also presented for comparison. To facilitate the work of plotting array beam patterns, the coordinate system used is $(U, V)$ instead of $(\theta, \phi)$ with $\sin (U)=\sin (\theta) \cos (\theta)$ and $\sin (V)=\sin (\theta) \sin (\phi)$, respectively.

Example 1: This example considers the case of $K=1$. Three signal sources with signal-to-noise (SNR) all equal to $0 \mathrm{~dB}$ are impinging on the array from direction angles $\left(\theta_{1}, \phi_{1}\right)=\left(85^{\circ}, 40^{\circ}\right),\left(\theta_{2}, \phi_{2}\right)=\left(0^{\circ}, 5^{\circ}\right)$, and $\left(\theta_{3}, \phi_{3}\right)=\left(25^{\circ}, 40^{\circ}\right)$, respectively. Assume that the specified signal is the first signal with $f_{1}=1$ and that the others are the jammers. Moreover, the signals with direction angles 


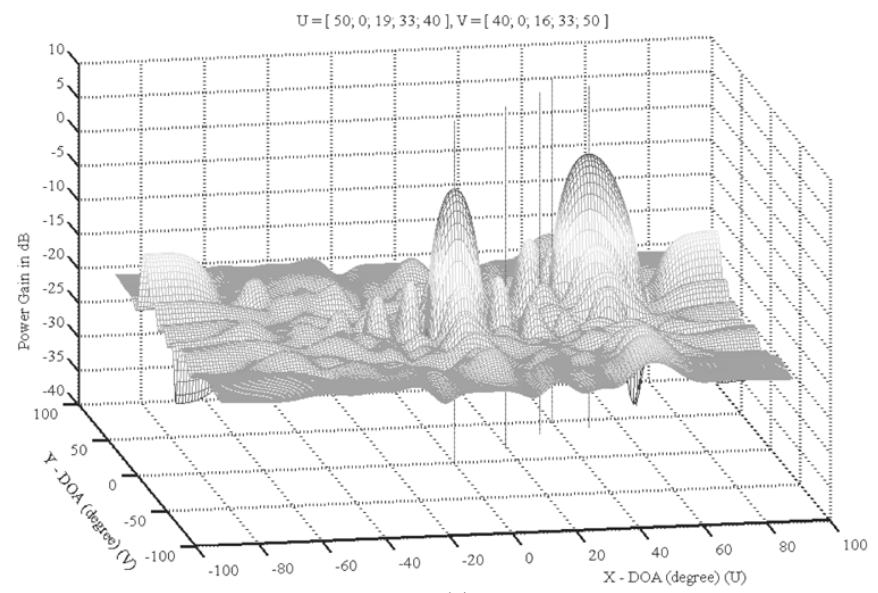

(a)

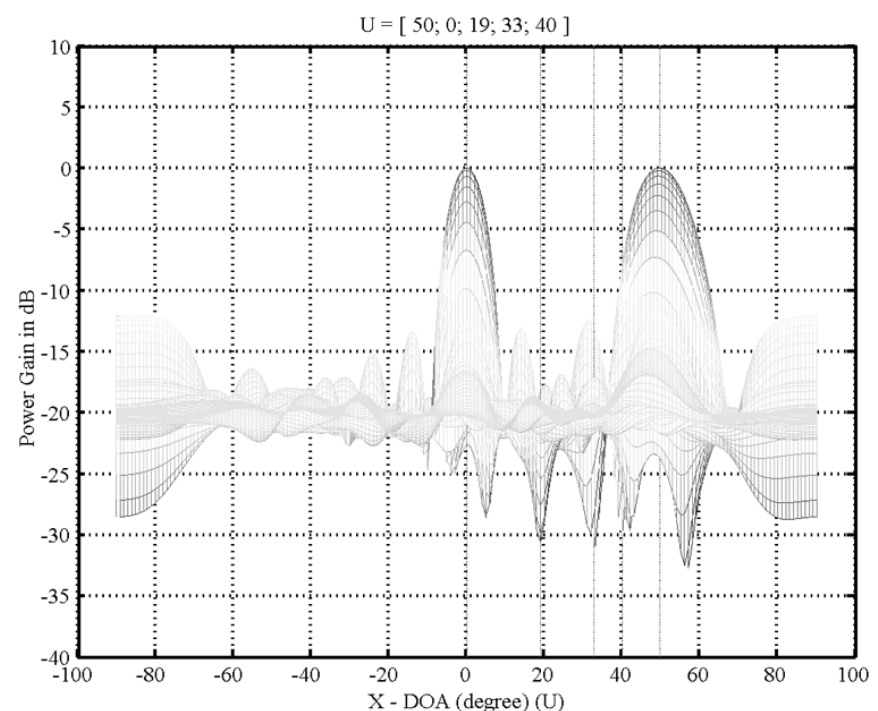

(c)

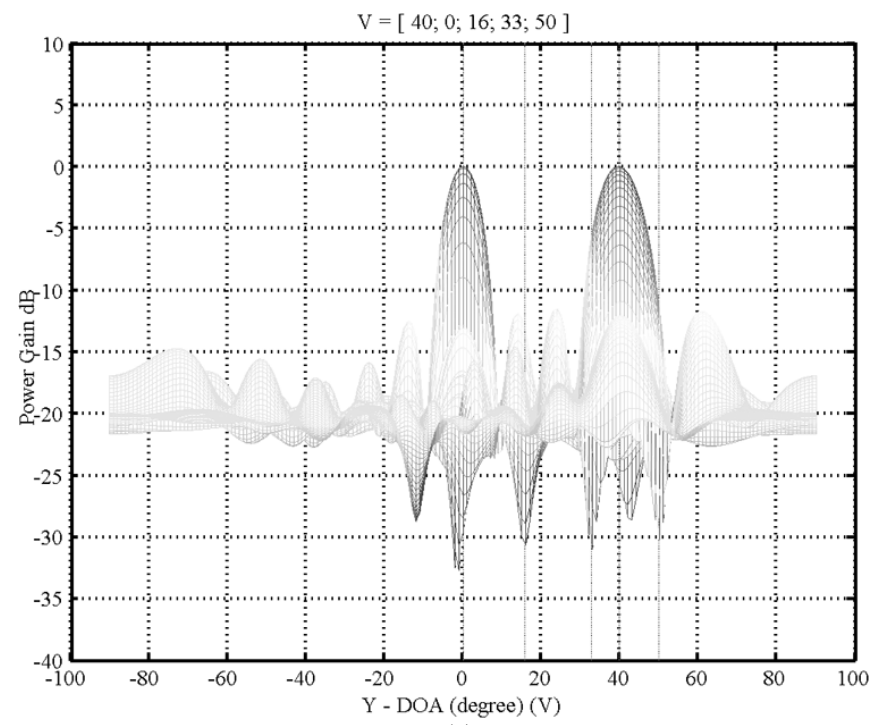

(c)

Fig. 9. (a) Two-dimensional array beam pattern without coherence for Example 2. (b) Beam pattern in the $U$ direction without coherence for Example 2. (c) Beam pattern in the $V$ direction without coherence for Example 2.

$\left(\theta_{1}, \phi_{1}\right)=\left(85^{\circ}, 40^{\circ}\right)$ and $\left(\theta_{3}, \phi_{3}\right)=\left(25^{\circ}, 40^{\circ}\right)$ are coherent. $M$ and $N$ are set to 11 . The subarray size for performing 2-D SS and 2-D WSS is set to $M_{s}=N_{s}=10$. Accordingly, the

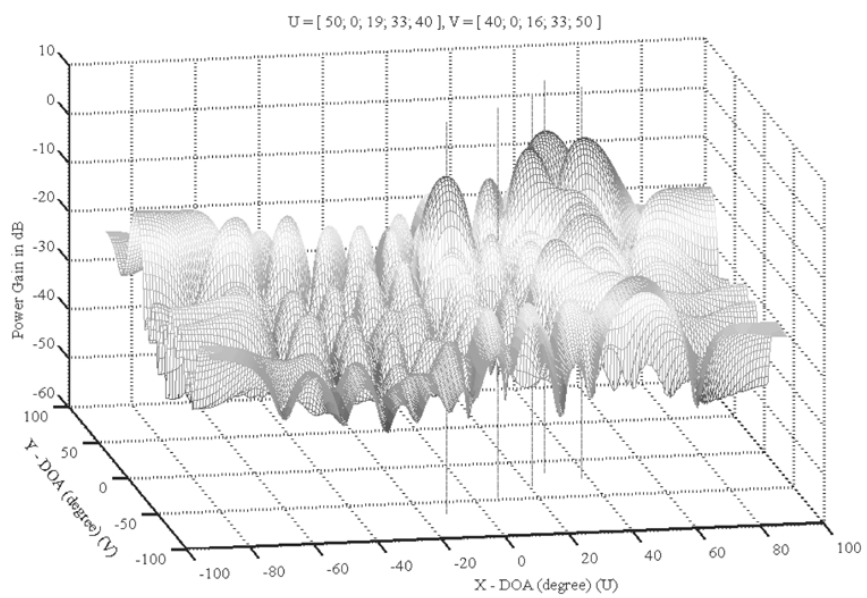

(a)

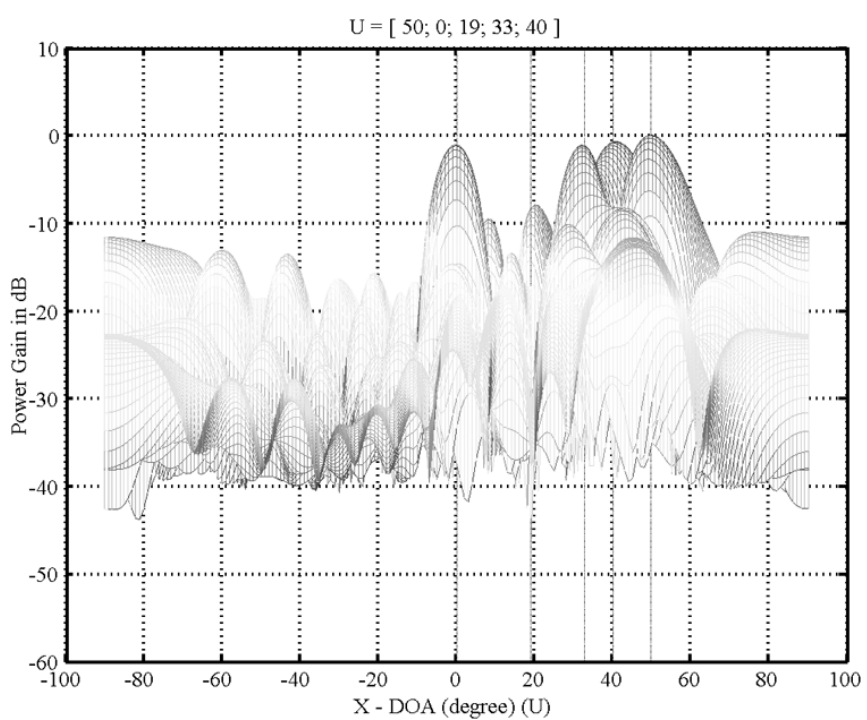

(b)

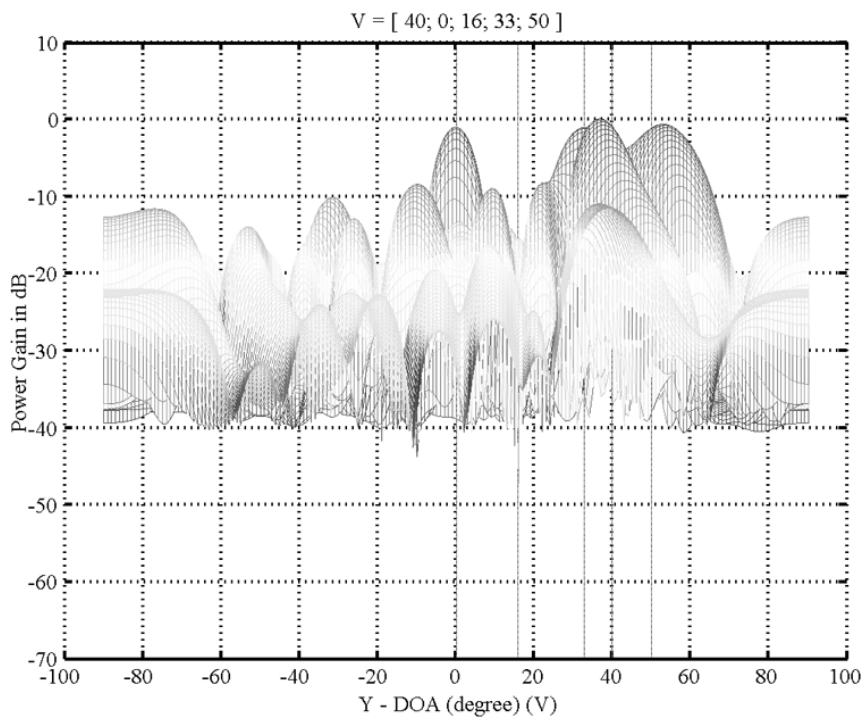

(c)

Fig. 10. (a) Two-dimensional array beam pattern with coherence for Example 2. (b) Beam pattern in the $U$ direction with coherence for Example 2. (c) Beam pattern in the $V$ direction with coherence for Example 2.

number of subarrays used is 4 . Figs. 4-7 plot the simulation results in terms of the array beam patterns, and Fig. 8 depicts 


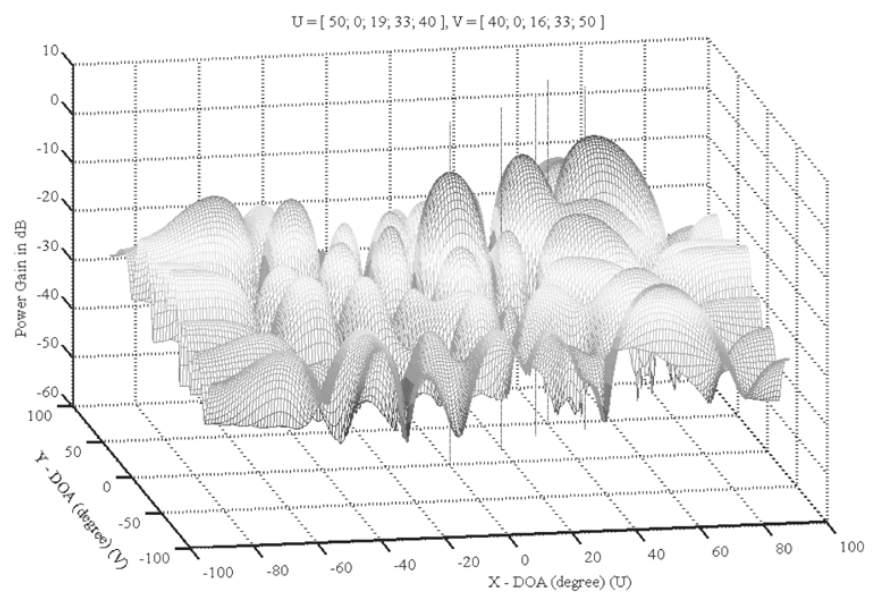

(a)

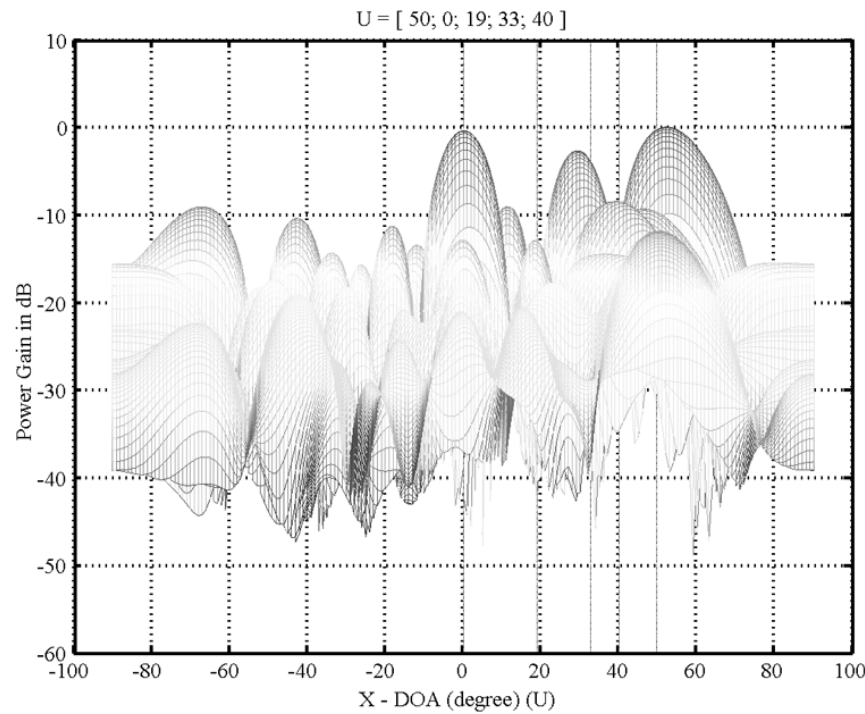

(b)

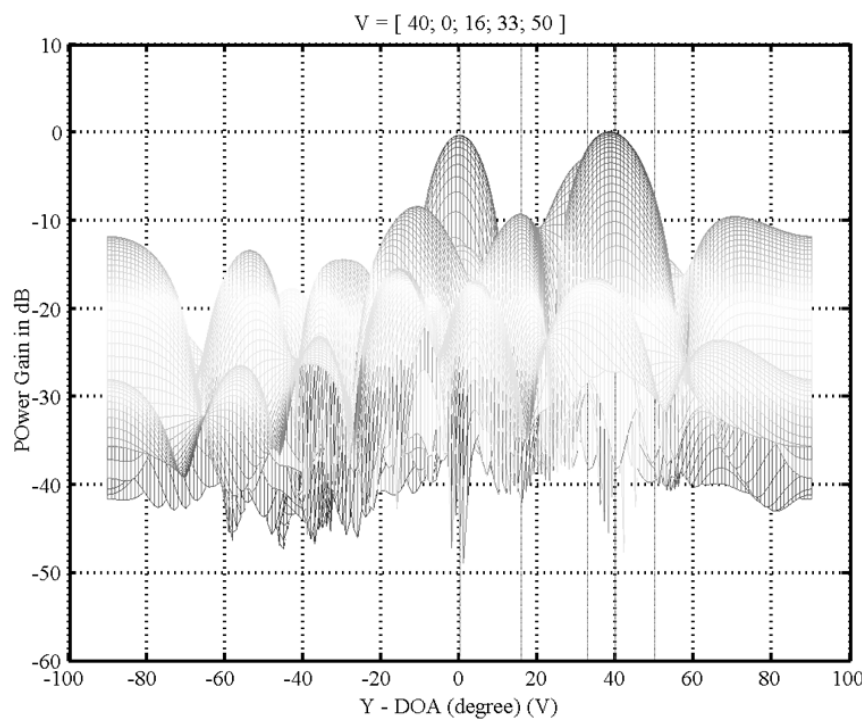

(c)

Fig. 11. (a) Two-dimensional array beam pattern after using 2-D SS for Example 2. (b) Beam pattern in the $U$ direction after using 2-D SS for Example 2. (c) Beam pattern in the $V$ direction after using 2-D SS for Example 2.

the corresponding array output signal-to-interference plus noise ratio (SINR) by utilizing the proposed GSC-based 2-D adaptive beamformer with conventional 2-D SS and with 2-D

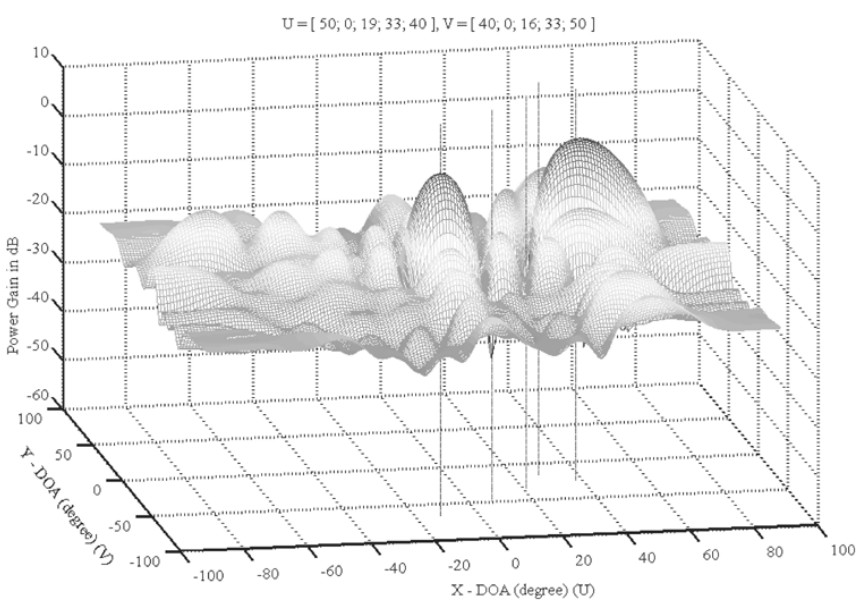

(a)

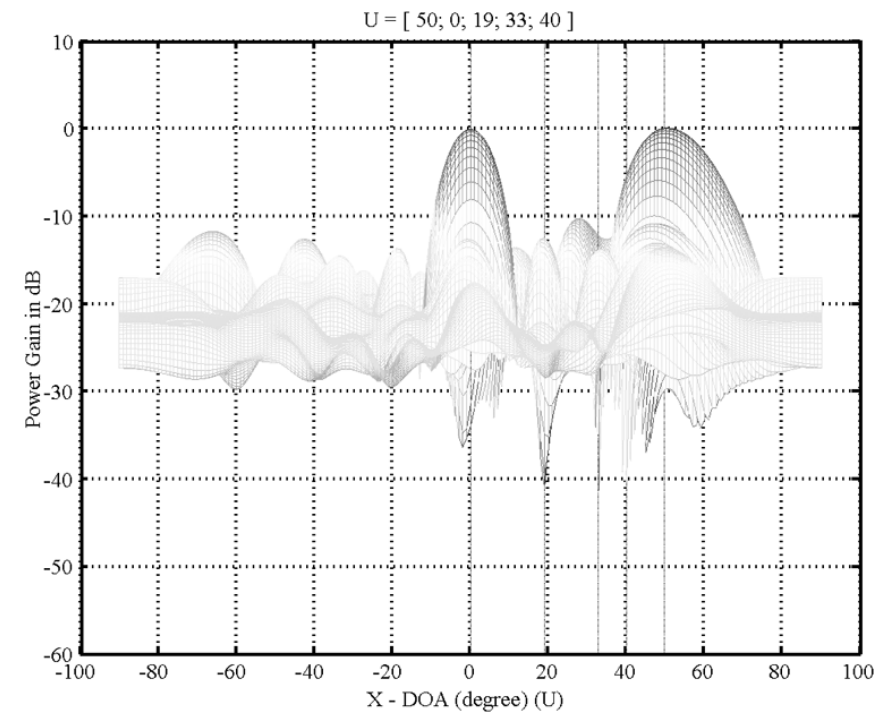

(b)

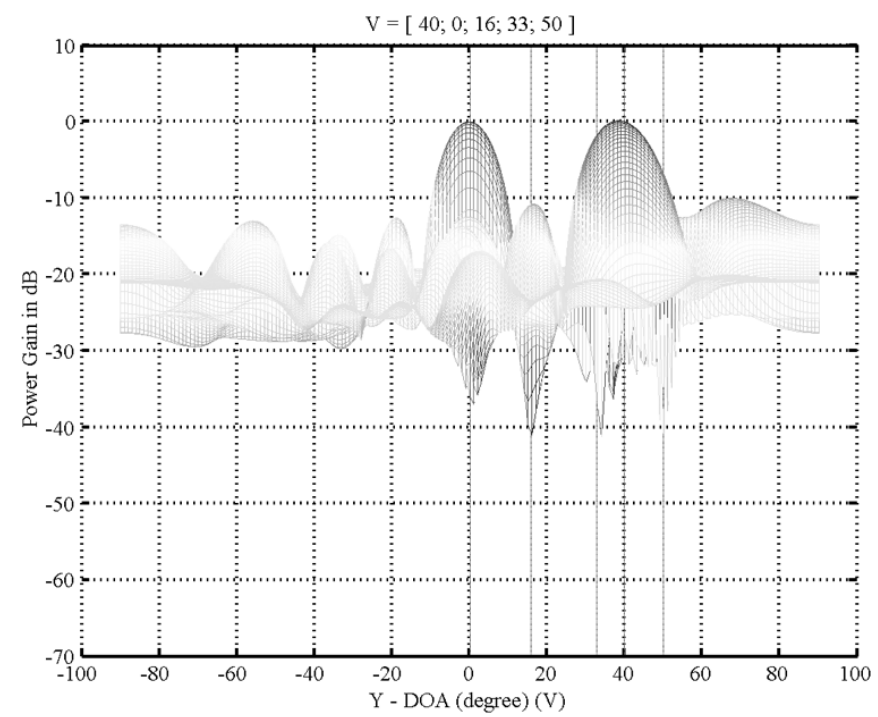

(c)

Fig. 12. (a) Two-dimensional array beam pattern after using 2-D WSS for Example 2. (b) Beam pattern in the $U$ direction after using 2-D WSS for Example 2. (c) Beam pattern in the $V$ direction after using 2-D WSS for Example 2.

WSS for comparison. The output SINRs obtained by using 30000 data snapshots for the results of using the 2-D WSS, 


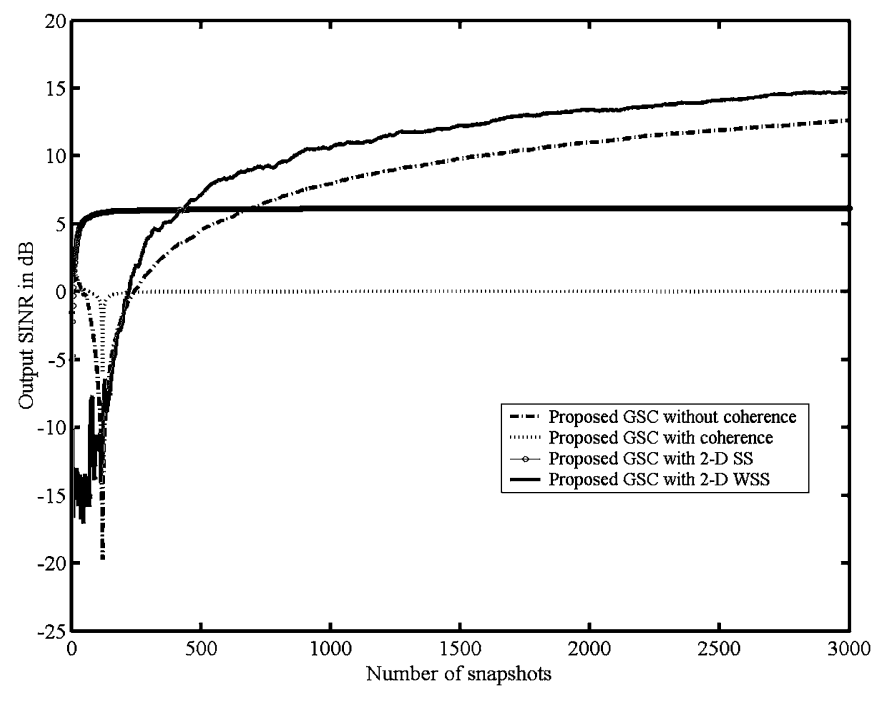

Fig. 13. Output SINR versus number of snapshots for Example 2.

TABLE III

OUTPUT SINRS (IN DECIBELS) OF USING DIFFERENT METHODS FOR EXAMPLE 2

\begin{tabular}{l|c|c|c|c}
\hline & $\begin{array}{l}\text { Without } \\
\text { Coherence }\end{array}$ & $\begin{array}{l}\text { With } \\
\text { Coherence }\end{array}$ & Using 2-D SS & $\begin{array}{l}\text { Using 2-D } \\
\text { WSS }\end{array}$ \\
\hline $\begin{array}{l}\text { Proposed } \\
\text { Method }\end{array}$ & 19.1078 & 0.0187517 & 6.17715 & 18.0475 \\
\hline $\begin{array}{l}\text { 2-D GSC } \\
\text { Method [7] }\end{array}$ & 18.8635 & 0.00253233 & 6.14786 & 17.9230 \\
\hline $\begin{array}{l}\text { LCMV } \\
\text { Method [11] }\end{array}$ & 18.8635 & 0.00253233 & 6.14786 & 17.9230 \\
\hline
\end{tabular}

the conventional 2-D SS, the result with coherence, and the result without coherence are listed in Table II. Three methods are presented for comparison: the proposed method, the 2-D conventional LCMV method that is a direct extension of the 1-D case [11], and the traditional 2-D GSC of [7]. We observe that the simulation results confirm the validity of the proposed method's capabilities for 2-D adaptive beamforming. The proposed method outperforms the traditional 2-D GSC [7] and the 2-D conventional LCMV method [11] in the main beam case.

Example 2: This example considers the case of $K=2$. Five signal sources with SNR all equal to $0 \mathrm{~dB}$ are impinging on the array from direction angels $\left(\theta_{1}, \phi_{1}\right)=\left(85^{\circ}, 40^{\circ}\right),\left(\theta_{2}, \phi_{2}\right)=$ $\left(0^{\circ}, 5^{\circ}\right),\left(\theta_{3}, \phi_{3}\right)=\left(25^{\circ}, 40^{\circ}\right),\left(\theta_{4}, \phi_{4}\right)=\left(50^{\circ}, 45^{\circ}\right)$, and $\left(\theta_{5}, \phi_{5}\right)=\left(88^{\circ}, 50^{\circ}\right)$, respectively. Assume that the specified signal is the first two signals with $f_{1}=f_{2}=1$, and the others are the jammers. Moreover, the signals with direction angles $\left(\theta_{1}, \phi_{1}\right)=\left(85^{\circ}, 40^{\circ}\right)$ and $\left(\theta_{4}, \phi_{4}\right)=\left(50^{\circ}, 45^{\circ}\right),\left(\theta_{2}, \phi_{2}\right)=$ $\left(0^{\circ}, 5^{\circ}\right)$ and $\left(\theta_{5}, \phi_{5}\right)=\left(88^{\circ}, 50^{\circ}\right)$ are coherent, respectively. $M$ and $N$ are set to 12 . The subarray size for performing 2-D SS and 2-D WSS is set to $M_{s}=N_{s}=9$. Accordingly, the number of subarrays used is 16 . Figs. 9-12 plot the simulation results in terms of the array beam patterns, and Fig. 13 depicts the corresponding array output SINR by utilizing the proposed GSC-based 2-D adaptive beamformer with conventional 2-D SS and with 2-D WSS for comparison. The output SINRs obtained by using 30000 data snapshots for the results of using the 2-D WSS, the conventional 2-D SS, the result with coherence, and the result without coherence are listed in Table III.
Again, three methods are presented for comparison: the proposed method, the 2-D conventional LCMV method that is a direct extension of the 1-D case [11], and the traditional 2-D GSC of [7]. Again, the simulation results confirm the validity of the proposed method's capabilities for 2-D adaptive beamforming. The proposed method outperforms the traditional 2-D GSC [7] and the 2-D conventional LCMV method [11] in the MBC case.

\section{CONCLUSION}

This paper has presented a method to construct a generalized sidelobe canceller (GSC) for performing 2-D adaptive array beamforming with multiple-beam constraints using a 2-D uniform planar array. Through the presented analytical formulas, the 2-D signal blocking matrix required by the 2-D GSC can be computed analytically without the need for complicated singular value decomposition. In the case with coherent signal sources, a 2-D weighted spatial smoothing scheme is proposed to effectively decorrelate the coherence between coherent signals, and hence, the performance degradation due to coherence can be alleviated. As compared with the existing 2-D spatial smoothing scheme, which is the most notable method for tackling coherent situation, several simulation examples have confirmed the validity of the theoretical works and shown that the proposed method can provide satisfactory performance.

\section{REFERENCES}

[1] L. J. Griffiths and C. W. Jim, "An alternative approach for linearly constrained adaptive beamforming," IEEE Trans. Antennas Propag., vol. AP-30, pp. 27-34, Jan. 1982.

[2] K. M. Buckley and L. J. Griffiths, "An adaptive generalized sidelobe canceller with derivative constraints," IEEE Trans. Antennas Propag., vol. AP-34, pp. 311-319, Mar. 1986.

[3] K. Takao and K. Uchida, "Beamspace partially adaptive antenna," Proc. Inst. Elect. Eng., H, Microwave Antenna Propag., vol. 136, pp. 439-444, Dec. 1989.

[4] B. D. Van Veen, "Eigenstructure based partially adaptive array design," IEEE Trans. Antennas Propag., vol. 36, no. 3, pp. 357-362, Mar. 1988.

[5] B. D. Van Veen and R. A. Roberts, "Partially adaptive beamformer design via output power minimization," IEEE Trans. Acoust., Speech, Signal Process., vol. ASSP-35, no. 11, pp. 1524-1532, Nov. 1987.

[6] S. -J. Yu and J. -H. Lee, "Design of partially adaptive beamformers based on information theoretic criteria," IEEE Trans. Antennas Propag., vol. 42, no. 5, pp. 676-689, May 1994.

[7] I. Salem, A. Hanafy, H. G. Hussein, and A. D. Moufid, "Two-dimensional generalized sidelobe canceller (GSC) with partial adaptivity," in Proc. Eighteenth Nat. Radio Science Conf., Mansoura, Egypt, Mar. 2001, pp. 81-88.

[8] J. T. Mayhan, "Area coverage adaptive nulling from geosynchronous satellites: Phased arrays versus multiple-beam antennas," IEEE Trans. Antennas Propag., vol. AP-34, pp. 410-419, Mar. 1986.

[9] K. -B. Yu, "Adaptive beamforming for satellite communication with selective earth coverage and jammer nulling capability," IEEE Trans. Signal Process., vol. 44, no. 12, pp. 3162-3166, Dec. 1996.

[10] J. -H. Lee and T. -F. Hsu, "Adaptive beamforming with multiple-beam constraints in the presence of coherent jammers," Signal Process., vol. 80, pp. 2475-2480, May 2000.

[11] O. L. Frost, "An algorithm for constrained adaptive array processing," Proc. IEEE, vol. 60, no. 8, pp. 926-935, Aug. 1972.

[12] A. Graham, Kronectker Products and Matrix Calculus With Applications. New York: Ellis Horwood, 1981.

[13] G. H. Golub and C. F. Van Loan, Matrix Computations, Second ed. Baltimore, MD: Johns Hopkins Univ. Press, 1989.

[14] C.-C. Yeh, J.-H. Lee, and Y.-M. Chen, "Estimating two-dimensional angles-of-arrival in coherent source environment," IEEE Trans. Acoust., Speech, Signal Process., vol. 37, no. 1, pp. 153-155, Jan. 1989. 
[15] T. J. Shan and T. Kailath, "Adaptive beamforming for coherent signals and interference," IEEE Trans. Acoust., Speech, Signal Process., vol. ASSP-33, no. 3, pp. 527-536, Jun. 1985.

[16] K. Takao and N. Kikuma, "An adaptive array utilizing adaptive spatial averaging technique for multipath environment," IEEE Trans. Antennas Propag., vol. AP-35, no. 12, pp. 1389-1395, Dec. 1987.

[17] K. C. Indukumar and V. U. Reddy, "Decorrelation of broadband signals by Toeplitz-block-Toeplitz structuring," IEEE Trans. Aerosp. Electron. Syst., vol. 30, no. 3, pp. 685-697, Jul. 1994.

[18] J. A. Cadzow, "Signal enhancement-A composite property mapping algorithm," IEEE Trans. Acoust., Speech, Signal Process., vol. 36, no. 1, pp. 49-62, Jan. 1988

[19] C. W. Jim and L. J. Griffiths, "Random Gain and Phase Error Effects in Opyimal Array Structures,” Dept. Elec. Eng., Univ. Colorado, Boulder, CO, Tech. Rep. EE 77-2, 1977.

[20] L. J. Griffiths and C. W. Jim, "A Generalized Sidelobe Canceling Structure for Adaptive Arrays," Signal Process. Lab., Dept. Elec. Eng., Univ. Colorado, Boulder, CO, Tech. Rep. SPL 78-2, 1978.

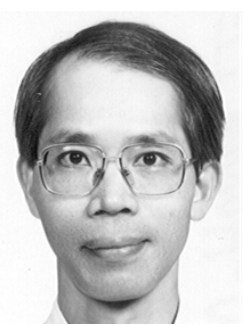

Ju-Hong Lee was born in I-Lan, Taiwan, R.O.C., on December 7, 1952. He received the B.S. degree from the National Cheng-Kung University, Tainan, Taiwan, in 1975, the M.S. degree from the National Taiwan University (NTU), Taipei, in 1977, and the Ph.D. degree from Rensselaer Polytechnic Institute (RPI), Troy, NY, in 1984, all in electrical engineering.

From September 1980 to July 1984, he was a Research Assistant and was involved in research on multidimensional recursive digital filtering with the Department of Electrical, Computer, and Systems Engineering, RPI. From August 1984 to July 1986, he was a Visiting Associate Professor and, in August 1986, became an Associate Professor with the Department of Electrical Engineering, NTU, where, since August 1989, he has been a Professor. He was appointed Visiting Professor with the Department of Computer Science and Electrical Engineering, University of Maryland, Baltimore, during a sabbatical leave in 1996. His current research interests include multidimensional digital signal processing, image processing, detection and estimation theory, analysis and processing of joint vibration signals for the diagnosis of cartilage pathology, and adaptive signal processing and its applications in communications.

Dr. Lee received Outstanding Research Awards from the National Science Council (NSC) of Taiwan in 1988, 1989, and from 1991 to 1994, and Distinguished Research Awards from the NSC from 1998 to 2004.

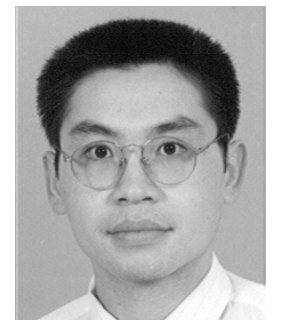

Yung-Han Lee was born in Taipei, Taiwan, R.O.C., on July 11, 1977. He received the B.S. degree in electrical engineering from the National Ocean University, Keelung, Taiwan, in 1999 and the M.S. degree from the Graduate Institute of Communication Engineering of National Taiwan University, Taipei, in 2004.

His current research interests include adaptive array signal processing and its applications in wireless communications. 